\title{
On the Fredholm-type theorems and sign properties of solutions for $(p, q)$-Laplace equations with two parameters
}

\author{
Vladimir Bobkov ${ }^{1}$ (D) Mieko Tanaka ${ }^{2}$
}

Received: 11 August 2018 / Accepted: 11 February 2019 / Published online: 19 February 2019 (c) Fondazione Annali di Matematica Pura ed Applicata and Springer-Verlag GmbH Germany, part of Springer Nature 2019

\begin{abstract}
We consider the Dirichlet problem for the nonhomogeneous equation $-\Delta_{p} u-\Delta_{q} u=$ $\alpha|u|^{p-2} u+\beta|u|^{q-2} u+f(x)$ in a bounded domain, where $p \neq q$, and $\alpha, \beta \in \mathbb{R}$ are parameters. We explore assumptions on $\alpha$ and $\beta$ that guarantee the resolvability of the considered problem. Moreover, we introduce several curves on the $(\alpha, \beta)$-plane allocating sets of parameters for which the problem has or does not have positive or sign-changing solutions, provided $f$ is of a constant sign.
\end{abstract}

Keywords $(p, q)$-Laplacian $\cdot$ Fredholm alternative $\cdot$ Existence of solutions $\cdot$ Positive solutions $\cdot$ Maximum principle $\cdot$ Linking method

Mathematics Subject Classification $35 \mathrm{~J} 62 \cdot 35 \mathrm{~J} 20 \cdot 35 \mathrm{P} 30 \cdot 35 \mathrm{~B} 50$

\section{Introduction and preliminaries}

Consider the boundary value problem

$$
\left\{\begin{aligned}
-\Delta_{p} u-\Delta_{q} u & =\alpha|u|^{p-2} u+\beta|u|^{q-2} u+f(x) & & \text { in } \Omega, \\
u & =0 & & \text { on } \partial \Omega,
\end{aligned}\right.
$$

where $\Delta_{r} u:=\operatorname{div}\left(|\nabla u|^{r-2} \nabla u\right)$ with $r=p$ or $r=q$ defines the $r$-Laplace operator, $p, q>1$ and, without loss of generality, $p>q$. Parameters $\alpha, \beta$ are real numbers, and $\Omega \subset \mathbb{R}^{N}$ is a bounded domain with $C^{2}$-boundary, $N \geq 1$. The source function $f$ belongs to $W^{-1, p^{\prime}}(\Omega)$, the dual of the Sobolev space $W_{0}^{1, p}:=W_{0}^{1, p}(\Omega), p^{\prime}=\frac{p}{p-1}$. The latter space

Vladimir Bobkov

bobkov@kma.zcu.cz

Mieko Tanaka

miekotanaka@rs.tus.ac.jp

1 Department of Mathematics and NTIS, Faculty of Applied Sciences, University of West Bohemia, Univerzitní 8, 30614 Plzeň, Czech Republic

2 Department of Mathematics, Tokyo University of Science, Kagurazaka 1-3, Shinjyuku-ku, Tokyo 162-8601, Japan 
is endowed with the norm $\|\nabla(\cdot)\|_{p}$, where $\|u\|_{p}:=\left(\int_{\Omega}|u|^{p} \mathrm{~d} x\right)^{1 / p}$ defines the norm of $L^{p}(\Omega)$.

The main "building block" of $\left(D_{\alpha, \beta, f}\right)$ is the nonlinear eigenvalue problem for the $r$ Laplacian

$$
\left\{\begin{aligned}
-\Delta_{r} u & =\lambda|u|^{r-2} u & & \text { in } \Omega, \\
u & =0 & & \text { on } \partial \Omega .
\end{aligned}\right.
$$

We say that $\lambda$ is an eigenvalue of the $r$-Laplacian if there exists a nonzero weak solution of $(1.0)_{r}$ called an eigenfunction associated with $\lambda$. Hereinafter, we denote by $\lambda_{1}(r)$ and $\varphi_{r}$ the first eigenvalue and an associated eigenfunction, respectively. It is known (see, e.g., [2,4]) that $\lambda_{1}(r)$ is simple and isolated, and it can be defined by

$$
\lambda_{1}(r)=\inf \left\{\frac{\|\nabla u\|_{r}^{r}}{\|u\|_{r}^{r}}: u \in W_{0}^{1, r} \backslash\{0\}\right\} .
$$

Moreover, $\varphi_{r} \in C^{1, \gamma}(\bar{\Omega})$ for some $\gamma \in(0,1)$ (see Remark 2.4 below), and we can choose $\varphi_{r}$ to be strictly positive [27]. By analogy with the linear case $r=2$, we will denote the set of all eigenvalues of the $r$-Laplacian as $\sigma\left(-\Delta_{r}\right)$ and the set of all eigenfunctions associated with the parameter $\lambda \in \mathbb{R}$ as $E S(r ; \lambda)$. For instance, $E S\left(r ; \lambda_{1}(r)\right) \equiv \mathbb{R} \varphi_{r}$, and $E S(r ; \lambda)=\{0\}$ provided $\lambda \notin \sigma\left(-\Delta_{r}\right)$.

If we let $p=q$ and $\alpha=\beta$, then, up to scaling, $\left(D_{\alpha, \beta, f}\right)$ turns to the problem

$$
\left\{\begin{aligned}
-\Delta_{p} u & =\lambda|u|^{p-2} u+f(x) & & \text { in } \Omega, \\
u & =0 & & \text { on } \partial \Omega,
\end{aligned}\right.
$$

which had been actively studied in the following two directions:

1. Existence and multiplicity of solutions. In fact, if $\lambda$ is not an eigenvalue of $-\Delta_{p}$, then the existence is well known, see, e.g., [21, Theorem 3.1, p. 60]. Moreover, in the linear case $p=2$ the complete information about the existence and multiplicity is provided via the Fredholm alternative. On the other hand, the situation is drastically different in the nonlinear case $p \neq 2$. It was investigated in $[12,13,28]$ (see also references therein), that the nontrivial multiplicity of solutions of (1.2) can occur as for $\lambda=\lambda_{1}(p)$, and for $\lambda$ from a punctured neighborhood of $\lambda_{1}(p)$, depending on assumptions on $f$. Notice that the investigations of (1.2) were carried out mainly in a neighborhood of $\lambda_{1}(p)$ due to the lack of description of $\sigma\left(-\Delta_{p}\right)$ at higher eigenvalues.

2. Sign properties of solutions. Suppose that $f \in L^{\infty}(\Omega) \backslash\{0\}$ and $f \geq 0$ a.e. in $\Omega$. Then, the well-known maximum principle states that any solution of (1.2) is positive provided $\lambda<\lambda_{1}(p)$. Moreover, any solution of (1.2) is either nonnegative or sign-changing for $\lambda>\lambda_{1}(p)$, see, e.g., [2, Theorem 2.1]. The latter result is strengthened by the antimaximum principle [11,19]: There exists $\lambda_{f}>\lambda_{1}(p)$ such that any solution of (1.2) is negative provided $\lambda \in\left(\lambda_{1}(p), \lambda_{f}\right)$.

The aim of the present paper is to obtain some basic results on the existence of solutions of $\left(D_{\alpha, \beta, f}\right)$, as well as on their sign properties. As in the case of the problem (1.2), it is clear that the investigation of $\left(D_{\alpha, \beta, f}\right)$ should be preceded by the study of the corresponding unperturbed problem

$$
\left\{\begin{aligned}
-\Delta_{p} u-\Delta_{q} u & =\alpha|u|^{p-2} u+\beta|u|^{q-2} u & & \text { in } \Omega, \\
u & =0 & & \text { on } \partial \Omega .
\end{aligned}\right.
$$


While the left- and right-hand sides of $\left(D_{\alpha, \beta, 0}\right)$ have the same "homogeneity", the structure of the solution set of $\left(D_{\alpha, \beta, 0}\right)$ strongly depends on the choice of the parameters $\alpha$ and $\beta$, see, e.g., [6-8,26,29], where the existence, multiplicity, and behavior of solutions of $\left(D_{\alpha, \beta, 0}\right)$ have been comprehensively studied. In fact, depending on $\alpha$ and $\beta,\left(D_{\alpha, \beta, 0}\right)$ demonstrates a behavior similar to the one for problems with convex-concave nonlinearities [3] and indefinite nonlinearities $[1,16]$, but with a more essential inclination to eigenvalue problems.

Let us recall several results from [6,8] about the existence and nonexistence of positive solutions of $\left(D_{\alpha, \beta, 0}\right)$, some of which will be used in the subsequent sections. In [6], the following family of critical points was introduced:

$$
\beta_{p s}(\alpha):=\sup _{u \in \operatorname{int} C_{0}^{1}(\bar{\Omega})_{+}} \inf _{\varphi \in C_{0}^{1}(\bar{\Omega})_{+} \backslash\{0\}} \mathcal{L}_{\alpha}(u ; \varphi) \quad \text { for } \alpha \geq \lambda_{1}(p) .
$$

Here, $\mathcal{L}_{\alpha}(u ; \varphi)$ is the so-called extended functional (see [17]) defined as

$$
\mathcal{L}_{\alpha}(u ; \varphi):=\frac{\int_{\Omega}|\nabla u|^{p-2} \nabla u \nabla \varphi \mathrm{d} x+\int_{\Omega}|\nabla u|^{q-2} \nabla u \nabla \varphi \mathrm{d} x-\alpha \int_{\Omega}|u|^{p-2} u \varphi \mathrm{d} x}{\int_{\Omega}|u|^{q-2} u \varphi \mathrm{d} x},
$$

and int $C_{0}^{1}(\bar{\Omega})_{+}$is the interior of the positive cone $C_{0}^{1}(\bar{\Omega})_{+}$of $C_{0}^{1}(\bar{\Omega})$ given by

$$
\text { int } C_{0}^{1}(\bar{\Omega})_{+}:=\left\{u \in C_{0}^{1}(\bar{\Omega}): u(x)>0 \text { for all } x \in \Omega, \frac{\partial u}{\partial v}(x)<0 \text { for all } x \in \partial \Omega\right\} \text {, }
$$

where $v$ is the outward unit normal vector to $\partial \Omega$. It was proved in [6, Theorem 2.2] that $\beta_{p s}(\alpha)$ is the threshold curve on the $(\alpha, \beta)$-plane which separates sets of the existence and nonexistence of positive solutions of $\left(D_{\alpha, \beta, 0}\right)$. Namely, if $\alpha>\lambda_{1}(p)$, then $\left(D_{\alpha, \beta, 0}\right)$ has a positive solution when $\beta<\beta_{p s}(\alpha)$, and $\left(D_{\alpha, \beta, 0}\right)$ does not have positive solutions when $\beta>\beta_{p s}(\alpha)$. Moreover, if $\alpha<\lambda_{1}(p)$, then $\left(D_{\alpha, \beta, 0}\right)$ possesses a solution if and only if $\beta>\lambda_{1}(q)$, see [6, Propositions 1 and 2]. Borderline cases were also studied, see, in particular, [6, Proposition 4] and [8, Proposition 3]. As for the properties of the curve $\beta_{p s}(\alpha)$, it is known that $\beta_{p s}\left(\lambda_{1}(p)\right) \geq \beta_{*}, \beta_{p s}(\alpha)$ is nonincreasing, and $\beta_{p s}(\alpha)=\lambda_{1}(q)$ for all $\alpha \geq \alpha_{*}$, where

$$
\alpha_{*}:=\frac{\left\|\nabla \varphi_{q}\right\|_{p}^{p}}{\left\|\varphi_{q}\right\|_{p}^{p}}, \quad \beta_{*}:=\frac{\left\|\nabla \varphi_{p}\right\|_{q}^{q}}{\left\|\varphi_{p}\right\|_{q}^{q}},
$$

see [6, Proposition 3] and [8, Proposition 3 (ii)]. Notice that $\alpha_{*}>\lambda_{1}(p)$ and $\beta_{*}>\lambda_{1}(q)$, since $\lambda_{1}(p)$ and $\lambda_{1}(q)$ are simple and have different eigenspaces, see [8, Proposition 13].

We also refer the interested reader to the works $[5,10,18,24]$ for existence results and qualitative properties of solutions of other types of problems driven by the $(p, q)$-Laplace operator.

\section{Main results}

In this section, we collect our main results. We group them according to the existence of solutions of $\left(D_{\alpha, \beta, f}\right)$ and their qualitative properties.

Hereinafter, we will use the notations

$$
H_{\alpha}(u):=\|\nabla u\|_{p}^{p}-\alpha\|u\|_{p}^{p} \quad \text { and } \quad G_{\beta}(u):=\|\nabla u\|_{q}^{q}-\beta\|u\|_{q}^{q},
$$

and 


$$
\langle f, u\rangle:=\int_{\Omega} f u \mathrm{~d} x
$$

for the dual pairing between $f \in W^{-1, p^{\prime}}(\Omega)$ and $u \in W_{0}^{1, p}$.

\subsection{Existence}

Theorem 2.1 Let $f \in W^{-1, p^{\prime}}(\Omega)$. Assume that $\alpha, \beta \in \mathbb{R}$ are such that one of the following assumptions is satisfied:

(i) $G_{\beta}(u)<0$ for all $u \in E S(p ; \alpha) \backslash\{0\}$;

(ii) $G_{\beta}(u)>0$ for all $u \in E S(p ; \alpha) \backslash\{0\}$.

Then, $\left(D_{\alpha, \beta, f}\right)$ has at least one solution.

In order to quantify assumptions (i) and (ii) of Theorem 2.1, we introduce the following two families of critical values:

$$
\begin{aligned}
& \underline{\beta}(\alpha):=\inf \left\{\frac{\|\nabla u\|_{q}^{q}}{\|u\|_{q}^{q}}: u \in E S(p ; \alpha) \backslash\{0\}\right\}, \\
& \bar{\beta}(\alpha):=\sup \left\{\frac{\|\nabla u\|_{q}^{q}}{\|u\|_{q}^{q}}: u \in E S(p ; \alpha) \backslash\{0\}\right\}, \quad \alpha \in \mathbb{R},
\end{aligned}
$$

and set $\beta(\alpha)=+\infty$ and $\bar{\beta}(\alpha)=-\infty$ for $\alpha \notin \sigma\left(-\Delta_{p}\right)$. Note that $\beta(\alpha), \bar{\beta}(\alpha) \in$ $\left[\lambda_{1}(q),+\infty\right)$ provided $\alpha \in \sigma\left(-\Delta_{p}\right)$, see [7, Lemma 3.6], and the simplicity of $\lambda_{1}(p)$ and $\lambda_{1}(q)$ yields

$$
\underline{\beta}\left(\lambda_{1}(p)\right)=\bar{\beta}\left(\lambda_{1}(p)\right)=\beta_{*}>\lambda_{1}(q),
$$

where $\beta_{*}$ is defined in (1.4) and the inequality follows from [8, Proposition 13]. Then, Theorem 2.1 can be reformulated as follows.

Theorem 2.2 Let $f \in W^{-1, p^{\prime}}(\Omega)$. Assume that $\alpha, \beta \in \mathbb{R}$ are such that either $\beta<\beta(\alpha)$ or $\beta>\bar{\beta}(\alpha)$. Then, $\left(D_{\alpha, \beta, f}\right)$ has at least one solution.

The equalities in (2.3) show that if $\alpha=\lambda_{1}(p)$, then Theorem 2.1 does not provide the existence for $\left(D_{\alpha, \beta, f}\right)$ only when $\beta=\beta_{*}$. We have the following result in this case.

Theorem 2.3 Let $\partial \Omega$ be connected if $N \geq 2$. Assume that $p>2 q, \alpha=\lambda_{1}(p)$ and $\beta=\beta_{*}$. If $f \in L^{2}(\Omega)$ is such that $\int_{\Omega} f \varphi_{p} \mathrm{~d} x=0$, then $\left(D_{\alpha, \beta, f}\right)$ has at least one solution.

Recalling $p>q$, let us remark that the term $-\Delta_{q} u-\beta|u|^{q-2} u$ in the problem $\left(D_{\alpha, \beta, f}\right)$ can be considered as a specific case of a lower-order perturbation of the $p$-Laplacian, and hence some results stated in this section have to be typical for a more general setting, too. In particular, the $(p, q)$-Laplacian can be seen as a $(p-1)$-quasihomogeneous operator in the sense of [21, Definition 2.1, p. 58], and hence, [21, Theorem 3.2, p. 73] implies the existence of a solution of $\left(D_{\alpha, \beta, f}\right)$ whenever $f \in W^{-1, p^{\prime}}(\Omega)$ and $\alpha \notin \sigma\left(-\Delta_{p}\right)$. Thus, our Theorems 2.1 and 2.3 provide an improvement of this existence result.

\subsection{Sign properties}

Let us start with the following two remarks about a regularity of solutions of $\left(D_{\alpha, \beta, f}\right)$ and further properties of nonnegative solutions of $\left(D_{\alpha, \beta, f}\right)$. 
Remark 2.4 Assume that $\left\{\alpha_{n}\right\}$ and $\left\{\beta_{n}\right\}$ are bounded, $\left\{f_{n}\right\} \subset L^{\infty}(\Omega)$ is such that $\left\{\left\|f_{n}\right\|_{\infty}\right\}$ is bounded, and $\left\{c_{n}\right\}$ is nonnegative and bounded. If $u_{n}$ is a (weak) solution of

$$
\left\{\begin{aligned}
-\Delta_{p} u-c_{n} \Delta_{q} u & =\alpha_{n}|u|^{p-2} u+\beta_{n}|u|^{q-2} u+f_{n}(x) & & \text { in } \Omega, \\
u & =0 & & \text { on } \partial \Omega,
\end{aligned}\right.
$$

and $\left\{u_{n}\right\}$ is bounded in $W_{0}^{1, p}$, then there exist $\gamma \in(0,1)$ and $M>0$ independent of $n \in \mathbb{N}$ such that $u_{n} \in C^{1, \gamma}(\bar{\Omega})$ and $\left\|u_{n}\right\|_{C^{1, \gamma}(\bar{\Omega})} \leq M$ for all $n$. Indeed, thanks to the boundedness assumptions, Moser's iteration process (see, e.g., [25, Appendix C]) implies that $\left\{u_{n}\right\}$ is bounded in $L^{\infty}(\Omega)$, and then regularity results [22] and [23, Theorem 1.7] yield the boundedness of $\left\{u_{n}\right\}$ in $C^{1, \gamma}(\bar{\Omega})$. In particular, if $f \in L^{\infty}(\Omega)$, then any solution of $\left(D_{\alpha, \beta, f}\right)$ belongs to $C^{1, \gamma}(\bar{\Omega})$ for some $\gamma \in(0,1)$. The same regularity holds true for any eigenfunction of the $r$-Laplacian, $r>1$.

Remark 2.5 If $f \in L^{\infty}(\Omega), f \geq 0$ a.e. in $\Omega$, and $u \geq 0$ is a nonzero nonnegative solution of $\left(D_{\alpha, \beta, f}\right)$, then $u>0$ in view of the maximum principle [27, Theorem 5.4.1]. Moreover, Remark 2.4 and the boundary point lemma [27, Theorem 5.5.1] yield $u \in \operatorname{int} C_{0}^{1}(\bar{\Omega})_{+}$. Analogously, $\varphi_{r} \in \operatorname{int} C_{0}^{1}(\bar{\Omega})_{+}$, where $\varphi_{r}$ is the first (nonnegative) eigenfunction of the $r$-Laplacian, $r>1$.

Assuming $f \in W^{-1, p^{\prime}}(\Omega)$ and $f \geq 0$ in the weak sense, we introduce the following family of critical values:

$$
\beta_{f}(\alpha):=\inf \left\{\Phi_{\alpha}^{+}(u): u \in \mathcal{B}^{+}(\alpha)\right\}, \quad \alpha \in \mathbb{R},
$$

where

$$
\begin{aligned}
& \Phi_{\alpha}^{+}(u):=\frac{\|\nabla u\|_{q}^{q}}{\|u\|_{q}^{q}}+\frac{p-1}{p-q}\left(\frac{p-q}{q-1}\right)^{\frac{q-1}{p-1}} \frac{\left(H_{\alpha}(u)\right)^{\frac{q-1}{p-1}}\langle f, u\rangle^{\frac{p-q}{p-1}}}{\|u\|_{q}^{q}}, \\
& \mathcal{B}^{+}(\alpha):=\left\{u \in W_{0}^{1, p} \backslash\{0\}: u \geq 0 \text { a.e. in } \Omega \text { and } H_{\alpha}(u) \geq 0\right\} .
\end{aligned}
$$

Clearly, $\beta_{f}(\alpha) \in\left[\lambda_{1}(q),+\infty\right)$ for any $\alpha \in \mathbb{R}$. In Lemma 5.4 below, we study some other properties of $\beta_{f}$. In particular, we show that $\beta_{f}(\alpha)>\lambda_{1}(q)$ if and only if $\alpha<\alpha_{*}$, provided $\left\langle f, \varphi_{q}\right\rangle>0$, where $\alpha_{*}$ is defined in (1.4).

Theorem 2.6 Let $f \in W^{-1, p^{\prime}}(\Omega) \backslash\{0\}$ and $f \geq 0$ in the weak sense. If $\alpha \leq \lambda_{1}(p)$ and $\beta<\beta_{f}(\alpha)$, then any solution of $\left(D_{\alpha, \beta, f}\right)$ is nonnegative. If, in addition, $f \in L^{\infty}(\Omega)$, then any solution of $\left(D_{\alpha, \beta, f}\right)$ belongs to int $C_{0}^{1}(\bar{\Omega})_{+}$.

The result of Theorem 2.6 can be complemented by the following dichotomy.

Proposition 2.7 Let $f \in L^{\infty}(\Omega) \backslash\{0\}$ and $f \geq 0$ a.e. in $\Omega$. Then, for every $\beta<\beta_{f}\left(\lambda_{1}(p)\right)$ there exists $\delta(\beta)>0$ such that for all $\alpha \in\left(\lambda_{1}(p), \lambda_{1}(p)+\delta(\beta)\right)$ any solution of $\left(D_{\alpha, \beta, f}\right)$ is either positive or negative. In particular, $\left(D_{\alpha, \beta, f}\right)$ has no sign-changing solutions.

By means of the critical curve $\beta_{p s}(\alpha)$ defined in (1.3), we give the following fact.

Proposition 2.8 Let $f \in L^{\infty}(\Omega) \backslash\{0\}$ and $f \geq 0$ a.e. in $\Omega$. Assume that $\alpha \geq \lambda_{1}(p)$ and $\beta>\beta_{p s}(\alpha)$. Then, $\left(D_{\alpha, \beta, f}\right)$ has no nonnegative solutions. That is, any solution of $\left(D_{\alpha, \beta, f}\right)$ is either nonpositive or sign-changing.

Recall that $\beta_{p s}(\alpha)=\lambda_{1}(q)$ for all $\alpha \geq \alpha_{*}$. In this case, Proposition 2.8 can be refined as follows. 


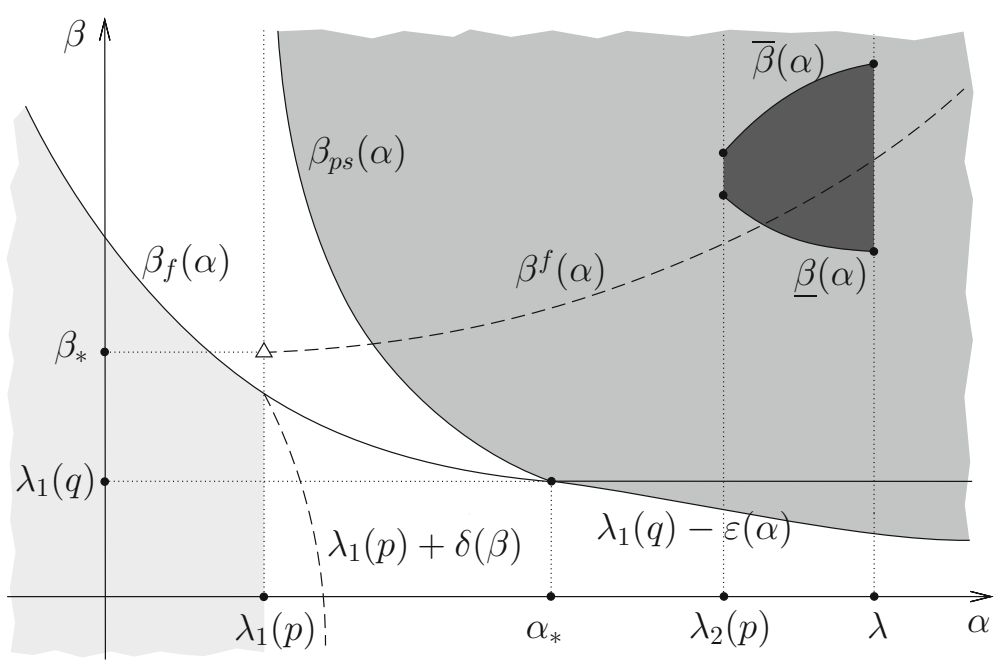

Fig. 1 Assume that $f \in L^{\infty}(\Omega) \backslash\{0\}, f \geq 0$ a.e. in $\Omega$, and $\left(\lambda_{2}(p), \lambda\right) \subset \sigma\left(-\Delta_{p}\right)$ for some $\lambda>\lambda_{2}(p)$. Light gray - any solution is positive. Gray—any solution is either nonpositive or sign-changing. Dark grayexistence is unknown

Proposition 2.9 Let $f \in L^{\infty}(\Omega) \backslash\{0\}$ and $f \geq 0$ a.e. in $\Omega$. Then, for any $\alpha>\alpha_{*}$ there exists $\varepsilon(\alpha)>0$ such that for any $\beta \geq \lambda_{1}(q)-\varepsilon(\alpha),\left(D_{\alpha, \beta, f}\right)$ has no nonnegative solutions.

We schematically depict the results of Theorems 2.2, 2.6, and Propositions 2.8, 2.9 in Fig. 1 .

To obtain additional qualitative properties of solutions of $\left(D_{\alpha, \beta, f}\right)$, we introduce one another family of critical values:

$$
\beta^{f}(\alpha):=\sup \left\{\Phi_{\alpha}^{-}(u): u \in \mathcal{B}^{-}(\alpha)\right\}, \quad \alpha \geq \lambda_{1}(p),
$$

where

$$
\begin{aligned}
& \Phi_{\alpha}^{-}(u):=\frac{\|\nabla u\|_{q}^{q}}{\|u\|_{q}^{q}}-\frac{p-1}{p-q}\left(\frac{p-q}{q-1}\right)^{\frac{q-1}{p-1}} \frac{\left(-H_{\alpha}(u)\right)^{\frac{q-1}{p-1}}\langle f, u\rangle^{\frac{p-q}{p-1}}}{\|u\|_{q}^{q}}, \\
& \mathcal{B}^{-}(\alpha):=\left\{u \in W_{0}^{1, p} \backslash\{0\}: u \geq 0 \text { a.e. in } \Omega \text { and } H_{\alpha}(u) \leq 0\right\} .
\end{aligned}
$$

We show in Lemma 5.4 below that $\beta^{f}(\alpha)<+\infty$ for all $\alpha \geq \lambda_{1}(p)$. Note that any function $u \in W_{0}^{1, p}$ can be decomposed as $u=u^{+}+u^{-}$, where $u^{+}:=\max \{u, 0\} \in W_{0}^{1, p}$ and $u^{-}:=\min \{u, 0\} \in W_{0}^{1, p}$.

Proposition 2.10 Let $f \in W^{-1, p^{\prime}}(\Omega) \backslash\{0\}$ and $f \geq 0$ in the weak sense. Assume that $\alpha>\lambda_{1}(p), \beta \in \mathbb{R}$, and let $u$ be a solution of $\left(D_{\alpha, \beta, f}\right)$. Then, the following assertions are satisfied:

(i) if $\beta<\beta_{f}(\alpha)$ and $u^{-} \not \equiv 0$, then $H_{\alpha}\left(u^{-}\right)<0$;

(ii) if $\beta>\beta^{f}(\alpha)$ and $u^{+} \not \equiv 0$, then $H_{\alpha}\left(u^{+}\right)>0$.

The rest of the article is organized as follows. In Sect. 3, we prove Theorem 2.1. In Sect. 4, we prove Theorem 2.3. Section 5 is devoted to the proof of the results stated in Sect. 2.2. 


\section{Proofs. Existence I}

In this section, we prove Theorem 2.1. We start by preparing several auxiliary results. We will use the sequence of eigenvalues $\left\{\lambda_{k}(p)\right\} \subset \sigma\left(-\Delta_{p}\right)$ introduced in [14] which can be defined as

$$
\lambda_{k}(p):=\inf _{h \in \mathscr{F}_{k}(p)} \max _{x \in S^{k-1}}\|\nabla h(x)\|_{p}^{p},
$$

where $S^{k-1}$ is the unit sphere in $\mathbb{R}^{k}, k \in \mathbb{N}$, and

$$
\begin{aligned}
\mathscr{F}_{k}(p) & :=\left\{h \in C\left(S^{k-1}, S(p)\right): h \text { is odd }\right\}, \\
S(p) & :=\left\{u \in W_{0}^{1, p}:\|u\|_{p}=1\right\} .
\end{aligned}
$$

It is known that $\lambda_{k}(p) \rightarrow+\infty$ as $k \rightarrow+\infty$, see [14, p. 195]. However, we recall that it is an open problem whether $\left\{\lambda_{k}(p)\right\}=\sigma\left(-\Delta_{p}\right)$, except in the cases $p=2$ and $N=1$, where the answer is affirmative.

Along this section, we assume that $f \in W^{-1, p^{\prime}}(\Omega)$, and we denote by $\|f\|_{*}$ the norm of $f$ in $W^{-1, p^{\prime}}(\Omega)$. Recall that weak solutions of $\left(D_{\alpha, \beta, f}\right)$ are critical points of the energy functional $E_{\alpha, \beta} \in C^{1}\left(W_{0}^{1, p}, \mathbb{R}\right)$ defined by

$$
E_{\alpha, \beta}(u)=\frac{1}{p} H_{\alpha}(u)+\frac{1}{q} G_{\beta}(u)-\langle f, u\rangle .
$$

To prove Theorem 2.1, we show that $E_{\alpha, \beta}$ has a linking structure provided $\alpha>\lambda_{1}(p)$ and $\alpha \neq \lambda_{k}(p), k \in \mathbb{N}$. Then, we can obtain a critical point of $E_{\alpha, \beta}$ whenever $E_{\alpha, \beta}$ satisfies the Palais-Smale condition.

Let us consider the set

$$
Y(\lambda):=\left\{u \in W_{0}^{1, p}:\|\nabla u\|_{p}^{p} \geq \lambda\|u\|_{p}^{p}\right\}, \quad \lambda \in \mathbb{R} .
$$

Hereinafter, $S_{+}^{k}$ stands for the closed unit upper hemisphere in $\mathbb{R}^{k+1}$ with the boundary $S^{k-1}$. We start by formulating the following linking lemma.

Lemma 3.1 ([7, Lemma 3.1]) Let $k \in \mathbb{N}$. Then, $h\left(S_{+}^{k}\right) \cap Y\left(\lambda_{k+1}(p)\right) \neq \emptyset$ for any $h \in$ $C\left(S_{+}^{k}, W_{0}^{1, p}\right)$ provided $\left.h\right|_{S^{k-1}}$ is odd.

Lemma 3.2 Let $\alpha, \beta \in \mathbb{R}$. If $\lambda>\max \{\alpha, 0\}$, then $E_{\alpha, \beta}$ is bounded from below and coercive on $Y(\lambda)$.

Proof Let $u \in Y(\lambda)$. We have

$$
E_{\alpha, \beta}(u) \geq \frac{1}{p}\left(1-\frac{\alpha}{\lambda}\right)\|\nabla u\|_{p}^{p}-\frac{\beta}{q\left(\lambda_{1}(p)\right)^{q / p}}|\Omega|^{\frac{p-q}{p}}\|\nabla u\|_{p}^{q}-\|f\|_{*}\|\nabla u\|_{p},
$$

where we used the Hölder and Poincaré inequalities to estimate the term $\|u\|_{q}^{q}$ of $E_{\alpha, \beta}(u)$. Since $p>q>1$ and $\lambda>\max \{\alpha, 0\}$, we easily deduce the desired boundedness from below and coercivity of $E_{\alpha, \beta}$ on $Y(\lambda)$.

Lemma 3.3 Let $\alpha, \beta \in \mathbb{R}$ be such that

$$
G_{\beta}(u) \neq 0 \text { for any } u \in E S(p ; \alpha) \backslash\{0\} .
$$

Then, $E_{\alpha, \beta}$ satisfies the Palais-Smale condition. 
Proof Let $\left\{u_{n}\right\} \subset W_{0}^{1, p}$ be a Palais-Smale sequence for $E_{\alpha, \beta}$. In view of the $\left(S_{+}\right)$-property of the operator $-\Delta_{p}-\Delta_{q}$ (see, e.g., [7, Remark 3.5]), it is sufficient to show the boundedness of $\left\{u_{n}\right\}$ in order to establish the desired Palais-Smale condition for $E_{\alpha, \beta}$. Suppose, by contradiction, that $\left\|\nabla u_{n}\right\|_{p} \rightarrow+\infty$ as $n \rightarrow+\infty$, up to a subsequence. Then, arguing in the same way as in [7, Lemma 3.3], we see that the sequence of normalized functions $v_{n}:=u_{n} /\left\|\nabla u_{n}\right\|_{p}$ converges strongly in $W_{0}^{1, p}$ (up to a subsequence) to some $v_{0} \in E S(p ; \alpha) \backslash\{0\}$. Hence, we get a contradiction whenever $\alpha \notin \sigma\left(-\Delta_{p}\right)$. On the other hand, if $\alpha \in \sigma\left(-\Delta_{p}\right)$, then

$o(1)=\frac{1}{\left\|\nabla u_{n}\right\|_{p}^{q}}\left(p E_{\alpha, \beta}\left(u_{n}\right)-\left\langle E_{\alpha, \beta}^{\prime}\left(u_{n}\right), u_{n}\right\rangle\right)=\left(\frac{p}{q}-1\right) G_{\beta}\left(v_{n}\right)-\frac{p-1}{\left\|\nabla u_{n}\right\|_{p}^{q-1}}\left\langle f, v_{n}\right\rangle$ as $n \rightarrow+\infty$. This yields $G_{\beta}\left(v_{0}\right)=0$, which contradicts (3.4).

We are now in a position to prove Theorem 2.1. The proof will be split into three cases, each of which is considered in a separate subsection.

\subsection{Case $\alpha \notin\left\{\lambda_{k}(p): k \in \mathbb{N}\right\}$}

First, we briefly handle the case $\alpha<\lambda_{1}(p)$. Since $Y\left(\lambda_{1}(p)\right)=W_{0}^{1, p}$, where $Y\left(\lambda_{1}(p)\right)$ is given by (3.3), Lemma 3.2 implies that $E_{\alpha, \beta}$ is bounded from below and coercive on $W_{0}^{1, p}$. Therefore, there exists a global minimizer of $E_{\alpha, \beta}$ which is a solution of $\left(D_{\alpha, \beta, f}\right)$.

Assume now that $\alpha>\lambda_{1}(p)$ and $\alpha \notin\left\{\lambda_{k}(p): k \in \mathbb{N}\right\}$. (Recall that the case $\alpha \in \sigma\left(-\Delta_{p}\right)$ is still possible.) Since $\lambda_{k}(p) \rightarrow+\infty$ as $k \rightarrow+\infty$, we can find $k \in \mathbb{N}$ such that $\lambda_{k}(p)<$ $\alpha<\lambda_{k+1}(p)$. Define

$$
\begin{aligned}
\gamma & :=\inf \left\{E_{\alpha, \beta}(u): u \in Y\left(\lambda_{k+1}(p)\right)\right\}>-\infty, \\
\Lambda & :=\left\{h \in C\left(S_{+}^{k}, W_{0}^{1, p}\right): \max _{x \in S^{k-1}} E_{\alpha, \beta}(h(x)) \leq \gamma-1 \text { and }\left.h\right|_{S^{k-1}} \text { is odd }\right\}, \\
c & :=\inf _{h \in \Lambda} \max _{x \in S_{+}^{k}} E_{\alpha, \beta}(h(x)),
\end{aligned}
$$

where $\gamma$ is well defined by Lemma 3.2. Note that if $\Lambda \neq \emptyset$, then Lemma 3.1 implies $c \geq \gamma$. Moreover, under assumption (i) or (ii) of the theorem, $E_{\alpha, \beta}$ satisfies the Palais-Smale condition by Lemma 3.3. Therefore, by the standard arguments via the deformation lemma we see that $c$ is a critical value of $E_{\alpha, \beta}$ provided $\Lambda \neq \emptyset$. Thus, let us construct an admissible map to show that $\Lambda \neq \emptyset$.

Let us choose some $0<\varepsilon<\left(\alpha-\lambda_{k}(p)\right) / 2$. Then, due to the definition of $\lambda_{k}(p)$, there exists $h_{0} \in \mathscr{F}_{k}(p)$ such that

$$
\max _{x \in S^{k-1}}\left\|\nabla h_{0}(x)\right\|_{p}^{p}<\lambda_{k}(p)+\varepsilon
$$

This implies

$$
\max _{x \in S^{k-1}} H_{\alpha}\left(h_{0}(x)\right)<\lambda_{k}(p)+\varepsilon-\alpha<-\varepsilon .
$$

Therefore, recalling that $p>q>1$, we can find a sufficiently large $T_{0}>0$ such that

$$
\max _{x \in S^{k-1}} E_{\alpha, \beta}\left(T h_{0}(x)\right)<-\frac{T^{p} \varepsilon}{p}+\frac{T^{q}}{q} \max _{x \in S^{k-1}} G_{\beta}\left(h_{0}(x)\right)+T\|f\|_{*}\left(\lambda_{k}(p)+\varepsilon\right)^{1 / p} \leq \gamma-1
$$


for all $T \geq T_{0}$. Using Dugundji's extension theorem [15], we may assume that $h_{0} \in$ $C\left(S_{+}^{k}, W_{0}^{1, p}\right)$, and hence $T_{0} h_{0} \in \Lambda$. Thus, $\Lambda \neq \emptyset$, which implies that $c$ is a critical value of $E_{\alpha, \beta}$.

\subsection{Case $\alpha \in\left\{\lambda_{k}(p): k \in \mathbb{N}\right\}$ under assumption (i)}

We may assume that $\lambda_{k}(p)=\alpha<\lambda_{k+1}(p)$ for some $k \in \mathbb{N}$. Let $\left\{\alpha_{n}\right\} \subset \mathbb{R}$ be a decreasing sequence satisfying

$$
\lambda_{k}(p)=\alpha<\alpha_{n}<\lambda_{k+1}(p) \text { for all } n \in \mathbb{N} \text {, and } \lim _{n \rightarrow+\infty} \alpha_{n}=\alpha .
$$

Similar to Sect. 3.1 above, consider for each $n \in \mathbb{N}$,

$$
\begin{aligned}
\gamma_{n} & :=\inf \left\{E_{\alpha_{n}, \beta}(u): u \in Y\left(\lambda_{k+1}(p)\right)\right\}>-\infty, \\
\Lambda_{n} & :=\left\{h \in C\left(S_{+}^{k}, W_{0}^{1, p}\right): \max _{x \in S^{k-1}} E_{\alpha_{n}, \beta}(h(x)) \leq \gamma_{n}-1 \text { and }\left.h\right|_{S^{k-1}} \text { is odd }\right\}, \\
c_{n} & :=\inf _{h \in \Lambda_{n}} \max _{x \in S_{+}^{k}} E_{\alpha_{n}, \beta}(h(x)) .
\end{aligned}
$$

Arguing as in Sect. 3.1, we get $\Lambda_{n} \neq \emptyset$, and hence, Lemma 3.1 yields $c_{n} \geq \gamma_{n}$. Moreover, since $\left\{\alpha_{n}\right\}$ is a decreasing sequence, we have $H_{\alpha_{n}}(u) \geq H_{\alpha_{1}}(u)$ for any $n \in \mathbb{N}$ and $u \in W_{0}^{1, p}$, whence

$$
c_{n} \geq \gamma_{n} \geq \inf \left\{E_{\alpha_{1}, \beta}(u): u \in Y\left(\lambda_{k+1}(p)\right)\right\}>-\infty,
$$

where the last inequality follows from Lemma 3.2. That is, $\left\{c_{n}\right\}$ is bounded from below.

Now, we claim that for any $n \in \mathbb{N}$ and $\varepsilon>0$ there exists $u_{n}^{\varepsilon} \in W_{0}^{1, p}$ such that

$$
\left|E_{\alpha_{n}, \beta}\left(u_{n}^{\varepsilon}\right)-c_{n}\right|<\varepsilon \text { and }\left\|E_{\alpha_{n}, \beta}^{\prime}\left(u_{n}^{\varepsilon}\right)\right\|_{*}<\varepsilon .
$$

Suppose, by contradiction, that there exist some $n \in \mathbb{N}$ and $\varepsilon>0$ such that

$$
\left.u \in E_{\alpha_{n}, \beta}^{-1}\left(\left[c_{n}-\varepsilon, c_{n}+\varepsilon\right]\right)\right) \text { implies }\left\|E_{\alpha_{n}, \beta}^{\prime}(u)\right\|_{*} \geq \varepsilon .
$$

(Clearly, the set $E_{\alpha_{n}, \beta}^{-1}\left(\left[c_{n}-\varepsilon, c_{n}+\varepsilon\right]\right)$ is nonempty.) Let us fix some $\varepsilon^{\prime} \in\left(0, \min \left\{\varepsilon, c_{n}-\right.\right.$ $\left.\left.\left(\gamma_{n}-1\right)\right\}\right)$. Then, using the deformation lemma (see, e.g., [9, Theorem 3.4 of Chapter I]), we can find a map $\eta \in C\left(W_{0}^{1, p}, W_{0}^{1, p}\right)$ which satisfies the following two assertions:

(a) $\eta(u)=u$ for any $u$ such that $u \notin E_{\alpha_{n}, \beta}^{-1}\left(\left[c_{n}-\varepsilon^{\prime}, c_{n}+\varepsilon^{\prime}\right]\right)$;

(b) $E_{\alpha_{n}, \beta}(\eta(u)) \leq c_{n}-\varepsilon^{\prime} / 2$ whenever $E_{\alpha_{n}, \beta}(u) \leq c_{n}+\varepsilon^{\prime} / 2$.

By the definition (3.7) of $c_{n}$, there exists $h \in \Lambda_{n}$ such that $\max _{x \in S_{+}^{k}} E_{\alpha_{n}, \beta}(h(x)) \leq c_{n}+\varepsilon^{\prime} / 2$. Since $h \in \Lambda_{n}$, we have $\max _{x \in S^{k-1}} E_{\alpha_{n}, \beta}(h(x)) \leq \gamma_{n}-1$. Thus, in view of the inequality $\gamma_{n}-1<c_{n}-\varepsilon^{\prime}$, Assertion (a) implies that $\eta(h(x))=h(x)$ for all $x \in S^{k-1}$, and hence $\eta \circ h \in \Lambda_{n}$. On the other hand, Assertion (b) yields $\max _{x \in S_{+}^{k}} E_{\alpha_{n}, \beta}(\eta(h(x))) \leq c_{n}-\varepsilon^{\prime} / 2$, which contradicts the definition of $c_{n}$. Consequently, the existence of $\left\{u_{n}^{\varepsilon}\right\} \subset W_{0}^{1, p}$ satisfying (3.9) is shown.

In view of (3.9), we can find for each $n \in \mathbb{N}$ a function $u_{n} \in W_{0}^{1, p}$ such that

$$
\left|E_{\alpha_{n}, \beta}\left(u_{n}\right)-c_{n}\right|<\frac{1}{n} \text { and }\left\|E_{\alpha_{n}, \beta}^{\prime}\left(u_{n}\right)\right\|_{*}<\frac{1}{n} .
$$


If $\left\|\nabla u_{n}\right\|_{p} \rightarrow+\infty$ as $n \rightarrow+\infty$, up to a subsequence, then by the same arguments as in the proof of [7, Lemma 3.3] the second inequality in (3.10) implies that the sequence of normalized functions $v_{n}:=u_{n} /\left\|\nabla u_{n}\right\|_{p}$ has a subsequence strongly convergent in $W_{0}^{1, p}$ to some $v_{0} \in E S(p ; \alpha) \backslash\{0\}$. Moreover, passing to the limit along this subsequence in

$$
\begin{aligned}
\frac{p\left(c_{n}-1\right)}{\left\|\nabla u_{n}\right\|_{p}^{q}}-\frac{1}{n\left\|\nabla u_{n}\right\|_{p}^{q-1}} & \leq \frac{1}{\left\|\nabla u_{n}\right\|_{p}^{q}}\left(p E_{\alpha_{n}, \beta}\left(u_{n}\right)-\left\langle E_{\alpha_{n}, \beta}^{\prime}\left(u_{n}\right), u_{n}\right\rangle\right) \\
& =\left(\frac{p}{q}-1\right) G_{\beta}\left(v_{n}\right)-\frac{p-1}{\left\|\nabla u_{n}\right\|_{p}^{q-1}}\left\langle f, v_{n}\right\rangle
\end{aligned}
$$

and recalling that $\left\{c_{n}\right\}$ is bounded from below [see (3.8)], we get $G_{\beta}\left(v_{0}\right) \geq 0$. However, this contradicts assumption (i) of the theorem. Therefore, $\left\{u_{n}\right\}$ is bounded in $W_{0}^{1, p}$ and, consequently, $\left\{c_{n}\right\}$ is also bounded. Furthermore, noting that

$$
\left\|E_{\alpha, \beta}^{\prime}\left(u_{n}\right)\right\|_{*} \leq\left\|E_{\alpha, \beta}^{\prime}\left(u_{n}\right)-E_{\alpha_{n}, \beta}^{\prime}\left(u_{n}\right)\right\|_{*}+\left\|E_{\alpha_{n}, \beta}^{\prime}\left(u_{n}\right)\right\|_{*} \leq \frac{\alpha_{n}-\alpha}{\lambda_{1}(p)^{1 / p}}\left\|u_{n}\right\|_{p}^{p-1}+\frac{1}{n},
$$

we obtain from (3.10) that $\left\{u_{n}\right\}$ is a (bounded) Palais-Smale sequence for $E_{\alpha, \beta}$. Consequently, $E_{\alpha, \beta}$ possesses a critical point since it satisfies the Palais-Smale condition by Lemma 3.3.

\subsection{Case $\alpha \in\left\{\lambda_{k}(p): k \in \mathbb{N}\right\}$ under assumption (ii)}

We may assume that $\lambda_{k}(p)<\alpha=\lambda_{k+1}(p)$ for some $k \in \mathbb{N}$. Let $\left\{\alpha_{n}\right\}$ be an increasing sequence satisfying

$$
\lambda_{k}(p)<\alpha_{n}<\alpha=\lambda_{k+1}(p) \text { for all } n \in \mathbb{N} \text {, and } \lim _{n \rightarrow+\infty} \alpha_{n}=\alpha .
$$

Let us define $\gamma_{n}, \Lambda_{n}$, and $c_{n}$ as in Sect. 3.2 above. By the same arguments as in Sect. 3.2, for any $n \in \mathbb{N}$ we can find $u_{n} \in W_{0}^{1, p}$ such that

$$
\left|E_{\alpha_{n}, \beta}\left(u_{n}\right)-c_{n}\right|<\frac{1}{n} \text { and }\left\|E_{\alpha_{n}, \beta}^{\prime}\left(u_{n}\right)\right\|_{*}<\frac{1}{n} .
$$

We claim that $\left\{c_{n}\right\}$ is bounded from above. If the claim is true, then, as in Sect. 3.2, the inequality

$$
\begin{aligned}
\frac{p\left(c_{n}+1\right)}{\left\|\nabla u_{n}\right\|_{p}^{q}}+\frac{1}{n\left\|\nabla u_{n}\right\|_{p}^{q-1}} & \geq \frac{1}{\left\|\nabla u_{n}\right\|_{p}^{q}}\left(p E_{\alpha_{n}, \beta}\left(u_{n}\right)-\left\langle E_{\alpha_{n}, \beta}^{\prime}\left(u_{n}\right), u_{n}\right\rangle\right) \\
& =\left(\frac{p}{q}-1\right) G_{\beta}\left(v_{n}\right)-\frac{p-1}{\left\|\nabla u_{n}\right\|_{p}^{q-1}}\left\langle f, v_{n}\right\rangle,
\end{aligned}
$$

in combination with assumption (ii) of the theorem, implies the boundedness of $\left\{u_{n}\right\}$ in $W_{0}^{1, p}$, which yields the existence of a critical point of $E_{\alpha, \beta}$.

Let us prove that $\left\{c_{n}\right\}$ is bounded from above. Note that $\Lambda_{n} \neq \emptyset$ for any $n \in \mathbb{N}$ by the same arguments as in Sect. 3.1. In particular, for $n=1$ we can find a map $h_{1} \in C\left(S_{+}^{k}, W_{0}^{1, p}\right)$ and a sufficiently large $T_{1}>0$ such that $\left.h_{1}\right|_{S^{k-1}}$ is odd, and

$$
\begin{aligned}
& \max _{x \in S^{k-1}} E_{\alpha_{1}, \beta}\left(T h_{1}(x)\right) \leq \gamma_{1}-1 \text { for any } T \geq T_{1}, \\
& \max _{x \in S^{k-1}} E_{\alpha_{1}, \beta}\left(T h_{1}(x)\right) \rightarrow-\infty \text { as } T \rightarrow+\infty
\end{aligned}
$$


see (3.6). Moreover, recalling that $\left\{\alpha_{n}\right\}$ is increasing, we have $H_{\alpha_{n}}(u) \leq H_{\alpha_{1}}(u)$ and hence $E_{\alpha_{n}, \beta}(u) \leq E_{\alpha_{1}, \beta}(u)$ for all $n \in \mathbb{N}$ and $u \in W_{0}^{1, p}$. Therefore, in view of (3.12), for any $n \in \mathbb{N}$ there exists a sufficiently large $T_{n}>0$ such that

$$
\max _{x \in S^{k-1}} E_{\alpha_{n}, \beta}\left(T_{n} h_{1}(x)\right) \leq \max _{x \in S^{k-1}} E_{\alpha_{1}, \beta}\left(T_{n} h_{1}(x)\right) \leq \gamma_{n}-1 .
$$

We may assume that $\left\{T_{n}\right\}$ is increasing.

Define a map $h_{n} \in C\left(S_{+}^{k}, W_{0}^{1, p}\right)$ for $n \geq 2$ by

$$
h_{n}(x):= \begin{cases}\left(2 x_{k+1} T_{1}+T_{n}\left(1-2 x_{k+1}\right)\right) h_{1}\left(\frac{x^{\prime}}{\sqrt{1-x_{k+1}^{2}}}, 0\right) & \text { if } 0 \leq x_{k+1} \leq \frac{1}{2}, \\ T_{1} h_{1}\left(\frac{2}{\sqrt{3}} x^{\prime}, \frac{2}{\sqrt{3}} \sqrt{x_{k+1}^{2}-\frac{1}{4}}\right) & \text { if } \frac{1}{2} \leq x_{k+1} \leq 1,\end{cases}
$$

where $x=\left(x_{1}, \cdots, x_{k+1}\right) \in S_{+}^{k}$ and $x^{\prime}=\left(x_{1}, \cdots, x_{k}\right)$. Considering $x_{k+1}=0$, we deduce from (3.13) that $h_{n} \in \Lambda_{n}$. Moreover, if $0 \leq x_{k+1} \leq \frac{1}{2}$, then

$$
E_{\alpha_{n}, \beta}\left(h_{n}(x)\right) \leq E_{\alpha_{1}, \beta}\left(h_{n}(x)\right) \leq \max _{T_{1} \leq T \leq T_{n}} \max _{x \in S^{k-1}} E_{\alpha_{1}, \beta}\left(T h_{1}(x)\right) \leq \gamma_{1}-1
$$

by (3.11), and if $\frac{1}{2} \leq x_{k+1} \leq 1$, then

$$
E_{\alpha_{n}, \beta}\left(h_{n}(x)\right) \leq \max _{x \in S_{+}^{k}} E_{\alpha_{n}, \beta}\left(T_{1} h_{1}(x)\right) \leq \max _{x \in S_{+}^{k}} E_{\alpha_{1}, \beta}\left(T_{1} h_{1}(x)\right)<+\infty .
$$

Therefore, since $c_{n} \leq \max _{x \in S_{+}^{k}} E_{\alpha_{n}, \beta}\left(h_{n}(x)\right)$ for each $n \in \mathbb{N}$, we conclude that $\left\{c_{n}\right\}$ is bounded from above, which finishes the proof of Theorem 2.1.

\section{Proofs. Existence II}

In this section, we prove Theorem 2.3. First, we provide the following auxiliary result. Let us decompose any $u \in L^{2}(\Omega)$ as $u=\gamma_{u} \varphi_{p}+u^{\perp}$, where $\varphi_{p}$ is the first eigenfunction of the $p$-Laplacian,

$$
\gamma_{u}:=\left\|\varphi_{p}\right\|_{2}^{-2} \int_{\Omega} u \varphi_{p} \mathrm{~d} x \text { and } \int_{\Omega} u^{\perp} \varphi_{p} \mathrm{~d} x=0 .
$$

It is clear that $u^{\perp}=u-\gamma_{u} \varphi_{p} \in W_{0}^{1, p}$ provided $u \in W_{0}^{1, p}$.

Lemma 4.1 Let $p \geq 2$. Assume that $\beta \in\left(\lambda_{1}(q), \beta_{*}\right]$. Then, there exists a constant $C>0$ such that for any $u \in W_{0}^{1, p}$ satisfying $G_{\beta}(u)<0$ there holds

$$
\left|G_{\beta}(u)\right| \leq C\left|\gamma_{u}\right|^{q-1}\left(\int_{\Omega}\left|\nabla \varphi_{p}\right|^{p-2}\left|\nabla u^{\perp}\right|^{2} \mathrm{~d} x\right)^{\frac{1}{2}}+C \int_{\Omega}\left|\nabla u^{\perp}\right|^{q} \mathrm{~d} x .
$$

If, in addition, $p \geq 2 q$ and $\gamma_{u} \neq 0$, then the following inequality is satisfied:

$$
\begin{aligned}
\left|G_{\beta}(u)\right| \leq & C\left|\gamma_{u}\right|^{-\frac{p-2 q}{2}}\left(\left|\gamma_{u}\right|^{p-2} \int_{\Omega}\left|\nabla \varphi_{p}\right|^{p-2}\left|\nabla u^{\perp}\right|^{2} \mathrm{~d} x+\int_{\Omega}\left|\nabla u^{\perp}\right|^{p} \mathrm{~d} x\right)^{\frac{1}{2}} \\
& +C \int_{\Omega}\left|u^{\perp}\right|^{q} \mathrm{~d} x .
\end{aligned}
$$


Proof Let us fix any $u=\gamma_{u} \varphi_{p}+u^{\perp} \in W_{0}^{1, p}$. Using the mean value theorem, we can find $\varepsilon \in(0,1)$ such that

$$
\begin{aligned}
0>G_{\beta}(u) & =\left|\gamma_{u}\right|^{q} G_{\beta}\left(\varphi_{p}\right)+\left\langle G_{\beta}^{\prime}\left(\gamma_{u} \varphi_{p}+\varepsilon u^{\perp}\right), u^{\perp}\right\rangle \geq\left\langle G_{\beta}^{\prime}\left(\gamma_{u} \varphi_{p}+\varepsilon u^{\perp}\right), u^{\perp}\right\rangle \\
& \geq-q \int_{\Omega}\left|\nabla\left(\gamma_{u} \varphi_{p}+\varepsilon u^{\perp}\right)\right|^{q-1}\left|\nabla u^{\perp}\right| \mathrm{d} x-\beta q \int_{\Omega}\left|\gamma_{u} \varphi_{p}+\varepsilon u^{\perp}\right|^{q-1}\left|u^{\perp}\right| \mathrm{d} x,
\end{aligned}
$$

where $G_{\beta}\left(\varphi_{p}\right) \geq 0$ follows from the assumption $\beta \in\left(\lambda_{1}(q), \beta_{*}\right]$.

During the proof, we will denote by $C>0$ various constants independent of $u$. To estimate (4.4), we develop an approach from the proof of [8, Proposition 11]. Let us start with the first summand in (4.4). Since $\varepsilon \in(0,1)$, we have

$$
\int_{\Omega}\left|\nabla\left(\gamma_{u} \varphi_{p}+\varepsilon u^{\perp}\right)\right|^{q-1}\left|\nabla u^{\perp}\right| \mathrm{d} x \leq \int_{\Omega}\left(\left|\gamma_{u}\right|\left|\nabla \varphi_{p}\right|+\left|\nabla u^{\perp}\right|\right)^{q-1}\left|\nabla u^{\perp}\right| \mathrm{d} x .
$$

Then, using Hölder's inequality, we get

$$
\begin{aligned}
& \int_{\Omega}\left(\left|\gamma_{u}\right|\left|\nabla \varphi_{p}\right|+\left|\nabla u^{\perp}\right|\right)^{q-1}\left|\nabla u^{\perp}\right| \mathrm{d} x \\
& \quad \leq C\left|\gamma_{u}\right|^{q-1} \int_{\Omega}\left|\nabla \varphi_{p}\right|^{q-1}\left|\nabla u^{\perp}\right| \mathrm{d} x+C \int_{\Omega}\left|\nabla u^{\perp}\right|^{q} \mathrm{~d} x \\
& \quad=C\left|\gamma_{u}\right|^{q-1} \int_{\Omega}\left|\nabla \varphi_{p}\right|^{\frac{p-2}{2}}\left|\nabla u^{\perp}\right| \cdot\left|\nabla \varphi_{p}\right|^{\frac{2 q-p}{2}} \mathrm{~d} x+C \int_{\Omega}\left|\nabla u^{\perp}\right|^{q} \mathrm{~d} x \\
& \quad \leq C\left|\gamma_{u}\right|^{q-1}\left(\int_{\Omega}\left|\nabla \varphi_{p}\right|^{p-2}\left|\nabla u^{\perp}\right|^{2} \mathrm{~d} x\right)^{\frac{1}{2}}\left(\int_{\Omega} \frac{\mathrm{d} x}{\left|\nabla \varphi_{p}\right|^{p-2 q}}\right)^{\frac{1}{2}}+C \int_{\Omega}\left|\nabla u^{\perp}\right|^{q} \mathrm{~d} x .
\end{aligned}
$$

Note that $\int_{\Omega}\left|\nabla \varphi_{p}\right|^{p-2}\left|\nabla u^{\perp}\right|^{2} \mathrm{~d} x<+\infty$, since $\varphi_{p} \in C^{1}(\bar{\Omega})$ (see Remark 2.4) and $p \geq 2$. Moreover, $\int_{\Omega} \frac{\mathrm{d} x}{\left|\nabla \varphi_{p}\right|^{p-2 q}}<+\infty$, too. Indeed, if $p \leq 2 q$, then this boundedness easily follows from the regularity $\varphi_{p} \in C^{1}(\bar{\Omega})$, while in the case $p>2 q$ the desired boundedness was discussed in [8, p. 1234].

On the other hand, if $p \geq 2 q$ and $\gamma_{u} \neq 0$, then, recalling that $\varepsilon \in(0,1)$ and using Hölder's inequality again, we can estimate the first summand in (4.4) as follows:

$$
\begin{aligned}
& \int_{\Omega}\left|\nabla\left(\gamma_{u} \varphi_{p}+\varepsilon u^{\perp}\right)\right|^{q-1}\left|\nabla u^{\perp}\right| \mathrm{d} x \leq \int_{\Omega}\left(\left|\gamma_{u}\right|\left|\nabla \varphi_{p}\right|+\left|\nabla u^{\perp}\right|\right)^{q-1}\left|\nabla u^{\perp}\right| \mathrm{d} x \\
& =\int_{\Omega}\left(\left|\gamma_{u}\right|\left|\nabla \varphi_{p}\right|+\left|\nabla u^{\perp}\right|\right)^{\frac{p-2}{2}}\left|\nabla u^{\perp}\right| \cdot\left(\left|\gamma_{u}\right|\left|\nabla \varphi_{p}\right|+\left|\nabla u^{\perp}\right|\right)^{\frac{2 q-p}{2}} \mathrm{~d} x \\
& \leq\left(\int_{\Omega}\left(\left|\gamma_{u}\right|\left|\nabla \varphi_{p}\right|+\left|\nabla u^{\perp}\right|\right)^{p-2}\left|\nabla u^{\perp}\right|^{2} \mathrm{~d} x\right)^{\frac{1}{2}}\left(\int_{\Omega} \frac{\mathrm{d} x}{\left(\left|\gamma_{u}\right|\left|\nabla \varphi_{p}\right|+\left|\nabla u^{\perp}\right|\right)^{p-2 q}}\right)^{\frac{1}{2}} \\
& \leq C\left(\left|\gamma_{u}\right|^{p-2} \int_{\Omega}\left|\nabla \varphi_{p}\right|^{p-2}\left|\nabla u^{\perp}\right|^{2} \mathrm{~d} x+\int_{\Omega}\left|\nabla u^{\perp}\right|^{p} \mathrm{~d} x\right)^{\frac{1}{2}} \frac{1}{\left|\gamma_{u}\right|^{\frac{p-2 q}{2}}}\left(\int_{\Omega} \frac{\mathrm{d} x}{\left|\nabla \varphi_{p}\right|^{p-2 q}}\right)^{\frac{1}{2}} .
\end{aligned}
$$


Let us now estimate the second term in (4.4). Since $\varepsilon \in(0,1)$ and $\left\|\varphi_{p}\right\|_{\infty}<+\infty$ (see Remark 2.4), we have

$$
\int_{\Omega}\left|\gamma_{u} \varphi_{p}+\varepsilon u^{\perp}\right|^{q-1}\left|u^{\perp}\right| \mathrm{d} x \leq C\left|\gamma_{u}\right|^{q-1} \int_{\Omega}\left|u^{\perp}\right| \mathrm{d} x+C \int_{\Omega}\left|u^{\perp}\right|^{q} \mathrm{~d} x .
$$

Thus, using Hölder's inequality and an embedding result of [20, Lemma 4.2], we get

$$
\begin{aligned}
\int_{\Omega}\left|\gamma_{u} \varphi_{p}+\varepsilon u^{\perp}\right|^{q-1}\left|u^{\perp}\right| \mathrm{d} x \leq & C\left|\gamma_{u}\right|^{-\frac{p-2 q}{2}}\left(\left|\gamma_{u}\right|^{p-2} \int_{\Omega}\left|\nabla \varphi_{p}\right|^{p-2}\left|\nabla u^{\perp}\right|^{2} \mathrm{~d} x\right)^{\frac{1}{2}} \\
& +C \int_{\Omega}\left|u^{\perp}\right|^{q} \mathrm{~d} x .
\end{aligned}
$$

Finally, combining (4.7) with (4.6), we obtain (4.3). To obtain (4.2), we apply the Sobolev embedding theorem to estimate the last summand in (4.7), and then combine (4.7) with (4.5).

Proof of Theorem 2.3 We will show that, under the imposed assumptions, $E_{\lambda_{1}(p), \beta_{*}}$ attains a global minimum. If $f \equiv 0$, then the existence of a global minimizer is given by [8, Theorem 2.6 (ii)]. Thus, we may assume that $f \not \equiv 0$. Let $\left\{u_{n}\right\} \subset W_{0}^{1, p}$ be a minimizing sequence for $E_{\lambda_{1}(p), \beta_{*}}$. It is not hard to see that each $E_{\lambda_{1}(p), \beta_{*}}\left(u_{n}\right)<0$. Indeed, fixing an arbitrary $u \in W_{0}^{1, p}$ satisfying $\langle f, u\rangle>0$, and recalling that $p>q>1$, we can find a sufficiently small $t>0$ such that

$$
E_{\lambda_{1}(p), \beta_{*}}(t u)=\frac{t^{p}}{p} H_{\lambda_{1}(p)}(u)+\frac{t^{q}}{q} G_{\beta_{*}}(u)-t\langle f, u\rangle<0 .
$$

Let us prove that $\left\{u_{n}\right\}$ is bounded in $W_{0}^{1, p}$. Suppose, by contradiction, that $\left\|\nabla u_{n}\right\|_{p} \rightarrow+\infty$ as $n \rightarrow+\infty$, up to a subsequence. Making the $L^{2}(\Omega)$-decomposition $u_{n}=\gamma_{n} \varphi_{p}+u_{n}^{\perp}$ [see (4.1)], we conclude that $\left|\gamma_{n}\right| \rightarrow+\infty$ or $\left\|\nabla u_{n}^{\perp}\right\|_{p} \rightarrow+\infty$ as $n \rightarrow+\infty$. To reach a contradiction, let us estimate $E_{\lambda_{1}(p), \beta_{*}}\left(u_{n}\right)$ from below. First, to estimate $H_{\lambda_{1}(p)}\left(u_{n}\right)$, we use the improved Poincaré inequality obtained in [20], which states that

$$
H_{\lambda_{1}(p)}\left(u_{n}\right) \geq C\left|\gamma_{n}\right|^{p-2} \int_{\Omega}\left|\nabla \varphi_{p}\right|^{p-2}\left|\nabla u_{n}^{\perp}\right|^{2} \mathrm{~d} x+C\left\|\nabla u_{n}^{\perp}\right\|_{p}^{p} .
$$

Hereinafter, $C>0$ is a constant independent of $n \in \mathbb{N}$. Second, to estimate $\left\langle f, u_{n}\right\rangle$, we recall that $f \in L^{2}(\Omega),\left\langle f, \varphi_{p}\right\rangle=0$, and $p>2 q>2$, which yields

$$
\left|\left\langle f, u_{n}\right\rangle\right|=\left|\int_{\Omega} f u_{n} \mathrm{~d} x\right|=\left|\int_{\Omega} f u_{n}^{\perp} \mathrm{d} x\right| \leq\|f\|_{2}\left\|u_{n}^{\perp}\right\|_{2} \leq C\|f\|_{2}\left\|\nabla u_{n}^{\perp}\right\|_{p} .
$$

Finally, in order to apply Lemma 4.1 for estimating $G_{\beta_{*}}\left(u_{n}\right)$, let us show that we may assume $G_{\beta_{*}}\left(u_{n}\right)<0$ for all $n \in \mathbb{N}$. Indeed, if $G_{\beta_{*}}\left(u_{n}\right) \geq 0$ for all $n$, up to a subsequence, then, using the estimates (4.8) and (4.9), we get

$$
E_{\lambda_{1}(p), \beta_{*}}\left(u_{n}\right) \geq C\left|\gamma_{n}\right|^{p-2} \int_{\Omega}\left|\nabla \varphi_{p}\right|^{p-2}\left|\nabla u_{n}^{\perp}\right|^{2} \mathrm{~d} x+C\left\|\nabla u_{n}^{\perp}\right\|_{p}^{p}-C\|f\|_{2}\left\|\nabla u_{n}^{\perp}\right\|_{p} .
$$

If $\left\|\nabla u_{n}^{\perp}\right\|_{p} \rightarrow+\infty$ as $n \rightarrow+\infty$, then $E_{\lambda_{1}(p), \beta_{*}}\left(u_{n}\right) \rightarrow+\infty$ regardless the behavior of $\left\{\gamma_{n}\right\}$, which contradicts the minimization property of $\left\{u_{n}\right\}$. Hence, $\left\{u_{n}^{\perp}\right\}$ is bounded in $W_{0}^{1, p}$. Therefore, since $\left\|\nabla u_{n}\right\|_{p} \rightarrow+\infty$ as $n \rightarrow+\infty$, we have $\left|\gamma_{n}\right| \rightarrow+\infty$. We see from (4.10) that if $\left|\gamma_{n}\right|^{p-2} \int_{\Omega}\left|\nabla \varphi_{p}\right|^{p-2}\left|\nabla u_{n}^{\perp}\right|^{2} \mathrm{~d} x \rightarrow+\infty$ as $n \rightarrow+\infty$, up to a subsequence, then $E_{\lambda_{1}(p), \beta_{*}}\left(u_{n}\right) \rightarrow+\infty$, which is again impossible. This implies 
$\int_{\Omega}\left|\nabla \varphi_{p}\right|^{p-2}\left|\nabla u_{n}^{\perp}\right|^{2} \mathrm{~d} x \rightarrow 0$. In view of the embedding result [20, Lemma 4.2], we get $\left\|u_{n}^{\perp}\right\|_{2} \rightarrow 0$, and hence, (4.9) yields $\left\langle f, u_{n}\right\rangle \rightarrow 0$. Thus, we conclude from (4.10) and the behavior of $\left\langle f, u_{n}\right\rangle$ that $E_{\lambda_{1}(p), \beta_{*}}\left(u_{n}\right) \geq 0+o(1)$ as $n \rightarrow+\infty$, which contradicts the minimization property of $\left\{u_{n}\right\}$ and the fact that $E_{\lambda_{1}(p), \beta_{*}}\left(u_{n}\right)<0$ for each $n \in \mathbb{N}$. Thus, $G_{\beta_{*}}\left(u_{n}\right)<0$ for all $n \in \mathbb{N}$.

Substituting now (4.8), (4.9), and the estimate (4.2) for $G_{\beta_{*}}\left(u_{n}\right)$ (combined with Hölder's inequality) into $E_{\lambda_{1}(p), \beta_{*}}\left(u_{n}\right)$, we get

$$
\begin{aligned}
E_{\lambda_{1}(p), \beta_{*}}\left(u_{n}\right) & \geq C\left|\gamma_{n}\right|^{p-2} \int_{\Omega}\left|\nabla \varphi_{p}\right|^{p-2}\left|\nabla u_{n}^{\perp}\right|^{2} \mathrm{~d} x+C\left\|\nabla u_{n}^{\perp}\right\|_{p}^{p} \\
& -C\left|\gamma_{n}\right|^{q-1}\left(\int_{\Omega}\left|\nabla \varphi_{p}\right|^{p-2}\left|\nabla u_{n}^{\perp}\right|^{2} \mathrm{~d} x\right)^{\frac{1}{2}}-C\left\|\nabla u_{n}^{\perp}\right\|_{p}^{q}-C\|f\|_{2}\left\|\nabla u_{n}^{\perp}\right\|_{p} .
\end{aligned}
$$

Let us consider the following three possible cases.

1. $\left\|\nabla u_{n}^{\perp}\right\|_{p} \rightarrow+\infty$ and $\left|\gamma_{n}\right| \rightarrow+\infty$ as $n \rightarrow+\infty$. Using Young's inequality, we have

$$
\begin{aligned}
\left|\gamma_{n}\right|^{q-1}\left(\int_{\Omega}\left|\nabla \varphi_{p}\right|^{p-2}\left|\nabla u_{n}^{\perp}\right|^{2} \mathrm{~d} x\right)^{\frac{1}{2}} & =\varepsilon\left|\gamma_{n}\right|^{\frac{p-2}{2}}\left(\int_{\Omega}\left|\nabla \varphi_{p}\right|^{p-2}\left|\nabla u_{n}^{\perp}\right|^{2} \mathrm{~d} x\right)^{\frac{1}{2}} \cdot \frac{1}{\varepsilon}\left|\gamma_{n}\right|^{\frac{2 q-p}{2}} \\
& \leq \varepsilon^{2}\left|\gamma_{n}\right|^{p-2} \int_{\Omega}\left|\nabla \varphi_{p}\right|^{p-2}\left|\nabla u_{n}^{\perp}\right|^{2} \mathrm{~d} x+\frac{1}{\varepsilon^{2}}\left|\gamma_{n}\right|^{2 q-p}
\end{aligned}
$$

for any $\varepsilon>0$. Substituting (4.12) into (4.11), we obtain

$$
\begin{aligned}
E_{\lambda_{1}(p), \beta_{*}}\left(u_{n}\right) & \geq\left(C-C \varepsilon^{2}\right)\left|\gamma_{n}\right|^{p-2} \int_{\Omega}\left|\nabla \varphi_{p}\right|^{p-2}\left|\nabla u_{n}^{\perp}\right|^{2} \mathrm{~d} x+C\left\|\nabla u_{n}^{\perp}\right\|_{p}^{p} \\
& -\frac{C}{\varepsilon^{2}}\left|\gamma_{n}\right|^{2 q-p}-C\left\|\nabla u_{n}^{\perp}\right\|_{p}^{q}-C\|f\|_{2}\left\|\nabla u_{n}^{\perp}\right\|_{p} .
\end{aligned}
$$

Taking $\varepsilon>0$ small enough and recalling that $p>2 q$, we easily conclude that $E_{\lambda_{1}(p), \beta_{*}}\left(u_{n}\right) \rightarrow+\infty$ as $n \rightarrow+\infty$, which is impossible since $\left\{u_{n}\right\}$ is a minimizing sequence.

2. $\left\|\nabla u_{n}^{\perp}\right\|_{p} \rightarrow+\infty$ as $n \rightarrow+\infty$ and $\left\{\gamma_{n}\right\}$ is bounded. Since $\varphi_{p} \in C^{1}(\bar{\Omega})$ (see Remark 2.4) and $p>2$, we have

$$
\left|\gamma_{n}\right|^{q-1}\left(\int_{\Omega}\left|\nabla \varphi_{p}\right|^{p-2}\left|\nabla u_{n}^{\perp}\right|^{2} \mathrm{~d} x\right)^{\frac{1}{2}} \leq C\left|\gamma_{n}\right|^{q-1}\left\|\nabla u_{n}^{\perp}\right\|_{2} \leq C\left|\gamma_{n}\right|^{q-1}\left\|\nabla u_{n}^{\perp}\right\|_{p},
$$

and hence, recalling that $p>q>1$, we obtain from (4.11) that

$E_{\lambda_{1}(p), \beta_{*}}\left(u_{n}\right) \geq C\left\|\nabla u_{n}^{\perp}\right\|_{p}^{p}-C\left|\gamma_{n}\right|^{q-1}\left\|\nabla u_{n}^{\perp}\right\|_{p}-C\left\|\nabla u_{n}^{\perp}\right\|_{p}^{q}-C\|f\|_{2}\left\|\nabla u_{n}^{\perp}\right\|_{p} \rightarrow+\infty$ as $n \rightarrow+\infty$, a contradiction.

3. $\left\{\left\|\nabla u_{n}^{\perp}\right\|_{p}\right\}$ is bounded and $\left|\gamma_{n}\right| \rightarrow+\infty$ as $n \rightarrow+\infty$. In this case, $\left\{u_{n}^{\perp}\right\}$ converges weakly in $W_{0}^{1, p}$ and strongly in $L^{r}(\Omega), r \in\left(1, p^{*}\right)$, to some $u_{0}^{\perp} \in W_{0}^{1, p}$, up to a subsequence. Recalling that $p>2 q$, we see from the estimate (4.13) that if $\left|\gamma_{n}\right|^{p-2} \int_{\Omega}\left|\nabla \varphi_{p}\right|^{p-2}\left|\nabla u_{n}^{\perp}\right|^{2} \mathrm{~d} x \rightarrow+\infty$ as $n \rightarrow+\infty$, up to a subsequence, then $E_{\lambda_{1}(p), \beta_{*}}\left(u_{n}\right) \rightarrow+\infty$, which is impossible. This implies $\int_{\Omega}\left|\nabla \varphi_{p}\right|^{p-2}\left|\nabla u_{n}^{\perp}\right|^{2} \mathrm{~d} x \rightarrow 0$. In view of the embedding result [20, Lemma 4.2], we get $\left\|u_{n}^{\perp}\right\|_{2} \rightarrow 0$, which yields $u_{0}^{\perp} \equiv 0$ and hence $\left\|u_{n}^{\perp}\right\|_{q} \rightarrow 0$ due to the strong convergence of $\left\{u_{n}^{\perp}\right\}$ in $L^{r}(\Omega), r \in\left(1, p^{*}\right)$. Consequently, we see from (4.3) and (4.9) that

$$
\left|G_{\beta_{*}}\left(u_{n}\right)\right| \rightarrow 0 \text { and }\left|\left\langle f, u_{n}\right\rangle\right| \rightarrow 0 \text { as } n \rightarrow+\infty .
$$


Thus,

$$
E_{\lambda_{1}(p), \beta_{*}}\left(u_{n}\right)=H_{\lambda_{1}(p)}\left(u_{n}\right)+o(1) \geq o(1) \text { as } n \rightarrow+\infty,
$$

which contradicts the facts that each $E_{\lambda_{1}(p), \beta_{*}}\left(u_{n}\right)<0$ and $\left\{u_{n}\right\}$ is a minimizing sequence for $E_{\lambda_{1}(p), \beta_{*}}$.

Therefore, we conclude that $\left\{u_{n}\right\}$ is bounded in $W_{0}^{1, p}$, and hence $\inf _{W_{0}^{1, p}} E_{\lambda_{1}(p), \beta_{*}}=$ $E_{\lambda_{1}(p), \beta_{*}}\left(u_{n}\right)+o(1)>-\infty$. Moreover, since $E_{\lambda_{1}(p), \beta_{*}}$ is weakly lower-semicontinuous, we see that, up to a subsequence, $\left\{u_{n}\right\}$ converges strongly in $W_{0}^{1, p}$ to a global minimizer $u$ of $E_{\lambda_{1}(p), \beta_{*}}$, and hence, $u$ is a critical point of $E_{\lambda_{1}(p), \beta_{*}}$.

\section{Proofs. Sign properties}

In this section, we prove the results stated in Sect. 2.2. We start with the following auxiliary lemma which will be employed several times.

Lemma 5.1 Let $\alpha>\lambda_{1}(p)$. Assume that $u \in W_{0}^{1, p}$ satisfies $H_{\alpha}(u)<0$. Then, there exists a sequence $\left\{u_{n}\right\} \subset W_{0}^{1, p}$ such that $H_{\alpha}\left(u_{n}\right)=0$ for all $n \in \mathbb{N}$, and $u_{n} \rightarrow$ weakly in $W_{0}^{1, p}$ and strongly in $W_{0}^{1, q}$ as $n \rightarrow+\infty$. Moreover, if $u \geq 0$ a.e. in $\Omega$, then $\left\{u_{n}\right\}$ can be chosen such that $u_{n} \geq 0$ a.e. in $\Omega$ for all $n \in \mathbb{N}$.

Proof The claims can be obtained arguing in much the same way as in the proof of $[8$, Theorem 2.5 (ii)] by considering $u$ instead of $\varphi_{p}$.

Now, we provide several properties of the critical value $\beta_{f}(\alpha)$ defined by (2.4). Recall that the functional $\Phi_{\alpha}^{+}$and the set $\mathcal{B}^{+}(\alpha)$ are defined by (2.5) and (2.6), respectively.

Proposition 5.2 Let $f \in W^{-1, q^{\prime}}(\Omega) \backslash\{0\}$ and $f \geq 0$ in the weak sense. Let supp $f$ be the support of the distribution $f$. Then, the following assertions are satisfied:

(i) $\Phi_{\alpha}^{+}$is 0-homogeneous and $\mathcal{B}^{+}(\alpha) \neq \emptyset$ for all $\alpha \in \mathbb{R}$;

(ii) if $\alpha<\alpha_{*}$, then $\beta_{f}(\alpha) \geq \lambda_{1}(q)$, and $\beta_{f}(\alpha)>\lambda_{1}(q)$ if and only if $\left\langle f, \varphi_{q}\right\rangle>0$;

(iii) if $\alpha \geq \alpha_{*}$, then $\beta_{f}(\alpha)=\lambda_{1}(q)$;

(iv) if $\alpha \geq \lambda_{1}(p)$, then $\beta_{f}(\alpha) \leq \beta_{*}$;

(v) $\beta_{f}$ is nonincreasing and left-continuous;

(vi) if there is a nonempty open set $\widetilde{\Omega} \subset \Omega \backslash \operatorname{supp} f$, then $\sup \left\{\beta_{f}(\alpha): \alpha \leq \lambda_{1}(p)\right\}<+\infty$.

If, in addition, $\langle f, v\rangle>0$ for any $v \in W_{0}^{1, q} \backslash\{0\}$ with $v \geq 0$ a.e. in $\Omega$, then the following assertions are satisfied:

(vii) $\beta_{f}$ is continuous in $\left(-\infty, \lambda_{1}(p)\right)$;

(viii) $\beta_{f}(\alpha) \rightarrow+\infty$ as $\alpha \rightarrow-\infty$.

Proof Assertion (i). The 0-homogeneity of $\Phi_{\alpha}^{+}$is trivial. To obtain $\mathcal{B}^{+}(\alpha) \neq \emptyset$, we consider an eigenfunction $u$ of the $p$-Laplacian associated with some eigenvalue $\lambda_{k}(p)>\max \left\{\alpha, \lambda_{1}(p)\right\}$. Then, $u^{+} \not \equiv 0$ and $H_{\alpha}\left(u^{+}\right)=\left(\lambda_{k}(p)-\alpha\right)\left\|u^{+}\right\|_{p}^{p}>0$, which yields $u^{+} \in \mathcal{B}^{+}(\alpha)$.

Assertion (ii). The inequality $\beta_{f}(\alpha) \geq \lambda_{1}(q)$ is trivial. Moreover, if $\left\langle f, \varphi_{q}\right\rangle=0$, then $\beta_{f}(\alpha)=\lambda_{1}(q)$ by the definition (2.4) of $\beta_{f}(\alpha)$ since $\varphi_{q} \in \mathcal{B}^{+}(\alpha)$. Let $\left\langle f, \varphi_{q}\right\rangle>0$ and suppose, by contradiction, that there is some $\alpha<\alpha_{*}$ such that $\beta_{f}(\alpha)=\lambda_{1}(q)$. Then, in 
view of Assertion (i), we can find a minimizing sequence $\left\{u_{n}\right\} \subset \mathcal{B}^{+}(\alpha)$ for $\beta_{f}(\alpha)$ such that $\left\|u_{n}\right\|_{q}=1$ for all $n \in \mathbb{N}$,

$$
\left\|\nabla u_{n}\right\|_{q}^{q} \rightarrow \lambda_{1}(q) \text { and }\left(H_{\alpha}\left(u_{n}\right)\right)^{\frac{q-1}{p-1}}\left\langle f, u_{n}\right\rangle^{\frac{p-q}{p-1}} \rightarrow 0
$$

as $n \rightarrow+\infty$. Passing to a subsequence, we see that $u_{n} \rightarrow \varphi_{q}$ strongly in $W_{0}^{1, q}$ and $L^{q}(\Omega)$, where we assumed $\left\|\varphi_{q}\right\|_{q}=1$. Therefore,

$$
\lim _{n \rightarrow+\infty}\left\langle f, u_{n}\right\rangle=\left\langle f, \varphi_{q}\right\rangle>0,
$$

and hence, the second convergence in (5.1) implies $\lim _{n \rightarrow+\infty} H_{\alpha}\left(u_{n}\right)=0$. Let us show that $\left\{u_{n}\right\}$ is bounded in $W_{0}^{1, p}$. Indeed, if we suppose, by contradiction, that $\left\|\nabla u_{n}\right\|_{p} \rightarrow+\infty$ as $n \rightarrow+\infty$, up to a subsequence, then $\lim _{n \rightarrow+\infty} H_{\alpha}\left(u_{n}\right)=0$ implies $\left\|\nabla u_{n}\right\|_{p}^{p} \leq(\alpha+1)\left\|u_{n}\right\|_{p}^{p}$ for all sufficiently large $n \in \mathbb{N}$. Thus, according to [29, Lemma 9], there exists a constant $C=C(\alpha)>0$ independent of $n$ such that

$$
\left\|\nabla u_{n}\right\|_{p} \leq C\left\|u_{n}\right\|_{q}=C,
$$

which is a contradiction, and hence, $\left\{u_{n}\right\}$ is bounded in $W_{0}^{1, p}$. Recalling that $u_{n} \rightarrow \varphi_{q}$ strongly in $W_{0}^{1, q}$, we conclude from (5.2) that $u_{n} \rightarrow \varphi_{q}$ weakly in $W_{0}^{1, p}$, up to a subsequence, whence we get

$$
H_{\alpha}\left(\varphi_{q}\right) \leq \liminf _{n \rightarrow+\infty} H_{\alpha}\left(u_{n}\right)=0 .
$$

However, by the definition (1.4) of $\alpha_{*}$, (5.3) contradicts the assumption $\alpha<\alpha_{*}$.

Assertion (iii). Note that $\beta_{f}\left(\alpha_{*}\right)=\lambda_{1}(q)$ since $\varphi_{q} \in \mathcal{B}^{+}\left(\alpha_{*}\right)$. Thus, let us assume $\alpha>\alpha_{*}$. Then, $H_{\alpha}\left(\varphi_{q}\right)<0$, and hence, applying Lemma 5.1 to $\varphi_{q}$, we can find a sequence $\left\{u_{n}\right\} \subset W_{0}^{1, p} \backslash\{0\}$ such that $u_{n} \geq 0$ a.e. in $\Omega$ and $H_{\alpha}\left(u_{n}\right)=0$ for any $n \in \mathbb{N}$, and $\frac{\left\|\nabla u_{n}\right\|_{q}^{q}}{\left\|u_{n}\right\|_{q}^{q}} \rightarrow \lambda_{1}(q)$ as $n \rightarrow+\infty$. That is, $\left\{u_{n}\right\} \subset \mathcal{B}^{+}(\alpha)$, and the assertion follows.

Before proving Assertion (iv), let us establish Assertion (v). We start with the monotonicity of $\beta_{f}$. Suppose, by contradiction, that there exist $\alpha_{1}$ and $\alpha_{2}$ such that $\alpha_{1}<\alpha_{2}$ and $\beta_{f}\left(\alpha_{1}\right)<$ $\beta_{f}\left(\alpha_{2}\right)$. That is, we can find $u \in \mathcal{B}^{+}\left(\alpha_{1}\right)$ such that $\Phi_{\alpha_{1}}^{+}(u)<\beta_{f}\left(\alpha_{2}\right)$. If $H_{\alpha_{2}}(u) \geq 0$, then $u \in \mathcal{B}^{+}\left(\alpha_{2}\right)$. Moreover, $\alpha_{1}<\alpha_{2}$ implies $H_{\alpha_{1}}(u)>H_{\alpha_{2}}(u)$, and hence, $\Phi_{\alpha_{1}}^{+}(u) \geq \Phi_{\alpha_{2}}^{+}(u)$, which contradicts the definition of $\beta_{f}\left(\alpha_{2}\right)$. Therefore, $H_{\alpha_{2}}(u)<0$. Applying Lemma 5.1, we can find a sequence $\left\{u_{n}\right\} \subset W_{0}^{1, p}$ such that $u_{n} \geq 0$ a.e. in $\Omega$,

$$
H_{\alpha_{2}}\left(u_{n}\right)=0 \text { and } \Phi_{\alpha_{2}}^{+}\left(u_{n}\right)=\frac{\left\|\nabla u_{n}\right\|_{q}^{q}}{\left\|u_{n}\right\|_{q}^{q}} \rightarrow \frac{\|\nabla u\|_{q}^{q}}{\|u\|_{q}^{q}} \leq \Phi_{\alpha_{1}}^{+}(u) \text { as } n \rightarrow+\infty \text {. }
$$

We see that $\left\{u_{n}\right\} \subset \mathcal{B}^{+}\left(\alpha_{2}\right)$, and hence, (5.4) leads to a contradiction since $\Phi_{\alpha_{1}}^{+}(u)<\beta_{f}\left(\alpha_{2}\right)$.

Now we prove the left-continuity of $\beta_{f}$. Let us fix some $\alpha \in \mathbb{R}$ and consider a sequence $\left\{\alpha_{n}\right\}$ such that $\alpha_{n}<\alpha$ for all $n \in \mathbb{N}$ and $\lim _{n \rightarrow+\infty} \alpha_{n}=\alpha$. Since we know that $\liminf _{n \rightarrow+\infty} \beta_{f}\left(\alpha_{n}\right) \geq$ $\beta_{f}(\alpha)$ by the monotonicity obtained above, let us show that $\lim \sup \beta_{f}\left(\alpha_{n}\right) \leq \beta_{f}(\alpha)$. Arguing by contradiction, we suppose that, up to a subsequence,

$$
\delta:=\lim _{n \rightarrow+\infty} \beta_{f}\left(\alpha_{n}\right)>\beta_{f}(\alpha) .
$$

Thus, we can find $u \in \mathcal{B}^{+}(\alpha)$ satisfying $\Phi_{\alpha}^{+}(u)<\delta$. Since each $\alpha_{n}<\alpha$, we have $u \in \mathcal{B}^{+}\left(\alpha_{n}\right)$. Therefore, recalling that $\alpha_{n} \rightarrow \alpha$ as $n \rightarrow+\infty$, we get a contradiction by

$$
\delta=\lim _{n \rightarrow+\infty} \beta_{f}\left(\alpha_{n}\right) \leq \lim _{n \rightarrow+\infty} \Phi_{\alpha_{n}}^{+}(u)=\Phi_{\alpha}^{+}(u)<\delta .
$$


Assertion (iv) easily follows from Assertion (v) by noting that $\beta_{f}\left(\lambda_{1}(p)\right) \leq \beta_{*}$ in view of $\varphi_{p} \in \mathcal{B}^{+}\left(\lambda_{1}(p)\right)$.

Assertion (vi). Taking a nonnegative function $u \in C_{0}^{\infty}(\Omega) \backslash\{0\}$ such that supp $u \subset \widetilde{\Omega}$, we see that $H_{\alpha}(u) \geq 0$ for all $\alpha \leq \lambda_{1}(p)$ and $\langle f, u\rangle=0$, which yields the desired bound:

$$
\beta_{f}(\alpha) \leq \Phi_{\alpha}^{+}(u)=\frac{\|\nabla u\|_{q}^{q}}{\|u\|_{q}^{q}} \text { for any } \alpha \leq \lambda_{1}(p) .
$$

Assertion (vii). Let $\alpha<\lambda_{1}(p)$ and let $\left\{\alpha_{n}\right\}$ be an arbitrary sequence convergent to $\alpha$. Since we already know that $\beta_{f}$ is nonincreasing and left-continuous by Assertion (v), it is sufficient to assume that $\alpha<\alpha_{n}<\lambda_{1}(p)$ and to show that $\liminf _{n \rightarrow+\infty} \beta_{f}\left(\alpha_{n}\right) \geq \beta_{f}(\alpha)$. Suppose, by contradiction, that, up to a subsequence, $\lim _{n \rightarrow+\infty} \beta_{f}\left(\alpha_{n}\right)<\beta_{f}(\alpha)$. Thus, for any sufficiently large $n \in \mathbb{N}$ we can choose $u_{n} \in \mathcal{B}^{+}\left(\alpha_{n}\right)$ such that

$$
\beta_{f}\left(\alpha_{n}\right) \leq \Phi_{\alpha_{n}}^{+}\left(u_{n}\right) \leq \sup _{m} \Phi_{\alpha_{m}}^{+}\left(u_{m}\right)<\beta_{f}(\alpha),
$$

and we may assume $\left\|u_{n}\right\|_{q}=1$ for all $n$. The latter inequality in (5.5) implies the existence of $C>0$ such that

$$
\left\|\nabla u_{n}\right\|_{q}^{q} \leq C \text { and }\left(H_{\alpha_{n}}\left(u_{n}\right)\right)^{\frac{q-1}{p-1}}\left\langle f, u_{n}\right\rangle^{\frac{p-q}{p-1}} \leq C
$$

for all $n$. In view of the first bound in (5.6) and the choice $\left\|u_{n}\right\|_{q}=1$, we see that $\left\{u_{n}\right\}$ converges to some nonnegative function $u_{0} \in W_{0}^{1, q} \backslash\{0\}$ weakly in $W_{0}^{1, q}$ and strongly in $L^{q}(\Omega)$, up to a subsequence. By our assumption, we get $\lim _{n \rightarrow+\infty}\left\langle f, u_{n}\right\rangle=\left\langle f, u_{0}\right\rangle>0$, which implies the uniform boundedness of $H_{\alpha_{n}}\left(u_{n}\right)$ for all $n$. Hence, by the same argument as in Assertion (ii) [see (5.2)], we conclude that $\left\{u_{n}\right\}$ is bounded in $W_{0}^{1, p}$, and thereby it converges to $u_{0}$ weakly in $W_{0}^{1, p}$, up to a subsequence. Moreover, $H_{\alpha}\left(u_{0}\right) \geq 0$ by $\alpha \leq \lambda_{1}(p)$. Thus, we see that $u_{0} \in \mathcal{B}^{+}(\alpha)$ and we deduce from (5.5) that

$$
\Phi_{\alpha}^{+}\left(u_{0}\right) \leq \liminf _{n \rightarrow+\infty} \Phi_{\alpha_{n}}^{+}\left(u_{n}\right)<\beta_{f}(\alpha),
$$

which contradicts the definition of $\beta_{f}(\alpha)$.

Assertion (viii). Suppose, by contradiction, that $C:=\sup \left\{\beta_{f}(\alpha): \alpha \leq \lambda_{1}(p)\right\}<+\infty$. The monotonicity of $\beta_{f}$ [see Assertion (v)] implies the existence of a sequence $\left\{\alpha_{n}\right\}$ such that $\alpha_{n} \rightarrow-\infty$ and $\beta_{f}\left(\alpha_{n}\right) \rightarrow C$ as $n \rightarrow+\infty$. Therefore, by the definition of $\beta_{f}\left(\alpha_{n}\right)$, we can find a sequence $\left\{u_{n}\right\} \subset \mathcal{B}^{+}\left(\alpha_{n}\right)$ such that $\left\|u_{n}\right\|_{q}=1$ for each $n \in \mathbb{N}$ and $\Phi_{\alpha_{n}}^{+}\left(u_{n}\right) \leq C+1$. Arguing as in Assertion (vii) [see (5.6)], we obtain that $u_{n} \rightarrow u_{0} \not \equiv 0$ weakly in $W_{0}^{1, q}$ and strongly in $L^{q}(\Omega)$, up to a subsequence. Moreover, $H_{\alpha_{n}}\left(u_{n}\right)$ is uniformly bounded for all $n$. Since $\alpha_{n} \rightarrow-\infty$ as $n \rightarrow+\infty$, we conclude that $\left\{u_{n}\right\}$ is bounded in $W_{0}^{1, p}$ and $\left\|u_{n}\right\|_{p} \rightarrow 0$. However, this contradicts $u_{0} \not \equiv 0$.

The following result is crucial for the proof of Theorem 2.6 and Proposition 2.10 (ii).

Proposition 5.3 Let $f \in W^{-1, p^{\prime}}(\Omega)$ and $f \geq 0$ in the weak sense. Assume that $\alpha \in \mathbb{R}$ and $\beta<\beta_{f}(\alpha)$. If $u \in W_{0}^{1, p}$ is such that $u \geq 0$ a.e. in $\Omega$ and $H_{\alpha}(u)>0$, then

$$
H_{\alpha}(u)+G_{\beta}(u)+\langle f, u\rangle>0 .
$$

Proof If $u \in W_{0}^{1, p}$ is such that $u \geq 0$ a.e. in $\Omega$ and $H_{\alpha}(u)>0$, then $\langle f, u\rangle \geq 0$. Evidently, if $G_{\beta}(u) \geq 0$, then (5.7) is satisfied. Thus, let us assume that $G_{\beta}(u)<0$. Note that $u \in \mathcal{B}^{+}(\alpha)$, 


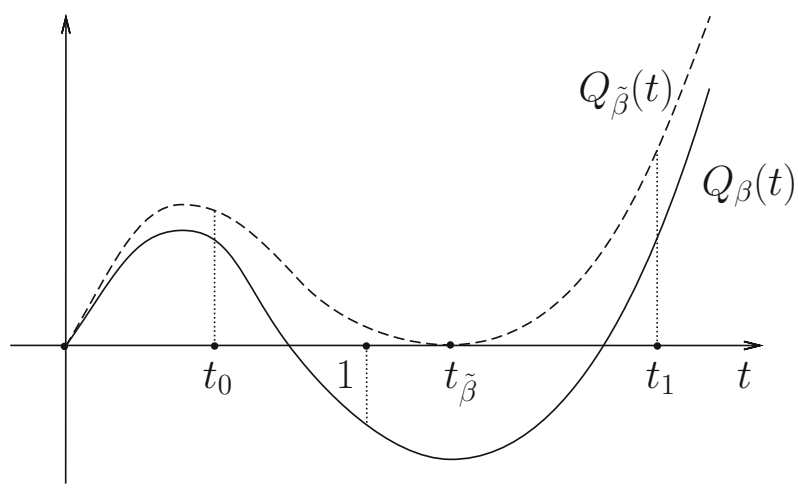

Fig. 2 Schematic behavior of $Q_{\beta}(t)$ and $Q_{\tilde{\beta}}(t)$

i.e., $u$ is admissible for the minimization problem (2.4) of $\beta_{f}(\alpha)$. Consequently, if $\langle f, u\rangle=0$, then $\beta<\beta_{f}(\alpha)$ implies $G_{\beta}(u)>0$, which is impossible. Therefore, $\langle f, u\rangle>0$.

Consider the function

$$
Q_{\beta}(t):=t^{p} H_{\alpha}(u)+t^{q} G_{\beta}(u)+t\langle f, u\rangle, \quad t \geq 0 .
$$

Since $p>q>1$, we see that $t^{p} H_{\alpha}(u)>0$ is the leading term at $t \rightarrow+\infty, t\langle f, u\rangle>0$ is the leading term at $t \rightarrow 0$, and $t^{q} G_{\beta}(u)<0$ has an impact in a middle range of $t$, see Fig. 2 .

If $Q_{\beta}(1)>0$, then (5.7) is satisfied. Thus, let us suppose that $Q_{\beta}(1) \leq 0$. In view of the behavior of $Q_{\beta}(t)$, we can find $t_{0}, t_{1}>0$ such that $t_{0}<1<t_{1}, Q_{\beta}\left(t_{0}\right)>0$, and $Q_{\beta}\left(t_{1}\right)>0$. Let us define the value

$$
q(b):=\min _{t_{0} \leq t \leq t_{1}} Q_{b}(t)
$$

and let $t_{b} \in\left(t_{0}, t_{1}\right)$ be a corresponding minimizer. We see that $q(\beta)=Q_{\beta}\left(t_{\beta}\right) \leq 0$ and $Q_{\beta}^{\prime}\left(t_{\beta}\right)=0$. Note that $Q_{b}(t)$ is strictly decreasing with respect to $b$ for any fixed $t>0$. Moreover, if $\hat{\beta}<\beta$ is such that $G_{\hat{\beta}}(u) \geq 0$, then $Q_{\hat{\beta}}(t) \geq q(\hat{\beta}) \geq c>0$ for some constant $c$ and all $t \geq t_{0}$. Therefore, noting that $q(\cdot)$ is continuous, we obtain the existence of $\tilde{\beta} \in(\hat{\beta}, \beta]$ such that

$$
Q_{\tilde{\beta}}\left(t_{\tilde{\beta}}\right)=0 \text { and } Q_{\tilde{\beta}}^{\prime}\left(t_{\tilde{\beta}}\right)=0 .
$$

Let us denote for simplicity $t=t_{\tilde{\beta}}$, and rewrite (5.8) as

$$
\left\{\begin{array}{l}
t^{p} H_{\alpha}(u)+t^{q} G_{\tilde{\beta}}(u)+t\langle f, u\rangle=0, \\
p t^{p} H_{\alpha}(u)+q t^{q} G_{\tilde{\beta}}(u)+t\langle f, u\rangle=0 .
\end{array}\right.
$$

Solving this system with respect to $G_{\tilde{\beta}}(u)$ and $t$, we obtain

$$
t=\left(\frac{q-1}{p-q}\right)^{\frac{1}{p-1}}\left(\frac{\langle f, u\rangle}{H_{\alpha}(u)}\right)^{\frac{1}{p-1}}
$$

and

$$
G_{\tilde{\beta}}(u)=-\frac{p-1}{p-q}\left(\frac{p-q}{q-1}\right)^{\frac{q-1}{p-1}}\left(H_{\alpha}(u)\right)^{\frac{q-1}{p-1}}\langle f, u\rangle^{\frac{p-q}{p-1}} .
$$


Expressing now $\tilde{\beta}$ from $G_{\tilde{\beta}}(u)$, we get

$$
\tilde{\beta}=\frac{\|\nabla u\|_{q}^{q}}{\|u\|_{q}^{q}}+\frac{p-1}{p-q}\left(\frac{p-q}{q-1}\right)^{\frac{q-1}{p-1}} \frac{\left(H_{\alpha}(u)\right)^{\frac{q-1}{p-1}}\langle f, u\rangle^{\frac{p-q}{p-1}}}{\|u\|_{q}^{q}} .
$$

However, this is impossible since $\tilde{\beta} \leq \beta<\beta_{f}(\alpha)$, and $u$ is an admissible function for the minimization formulation of $\beta_{f}(\alpha)$.

Proof of Theorem 2.6 Suppose that there is a solution $u$ of $\left(D_{\alpha, \beta, f}\right)$ with $\alpha \leq \lambda_{1}(p)$ and $\beta<\beta_{f}(\alpha)$ such that $u=u^{+}+u^{-}$and $u^{-} \not \equiv 0$. Then, $-u^{-} \geq 0$ and we have

$$
\left\langle E_{\alpha, \beta}^{\prime}(u), u^{-}\right\rangle=H_{\alpha}\left(-u^{-}\right)+G_{\beta}\left(-u^{-}\right)+\left\langle f,-u^{-}\right\rangle=0 .
$$

Thus, if $\alpha<\lambda_{1}(p)$, then $H_{\alpha}\left(-u^{-}\right)>0$, and hence, we get a contradiction to Proposition 5.3. In the case $\alpha=\lambda_{1}(p)$, it is enough to show that $u^{-} \notin \mathbb{R} \varphi_{p}$. Suppose, by contradiction and without loss of generality, that $-u^{-}=\varphi_{p}$. Then, we get

$$
\left\langle E_{\alpha, \beta}^{\prime}(u), u^{-}\right\rangle=G_{\beta}\left(\varphi_{p}\right)+\left\langle f, \varphi_{p}\right\rangle=0,
$$

and hence, $G_{\beta}\left(\varphi_{p}\right) \leq 0$. On the other hand, since $\beta<\beta_{f}\left(\lambda_{1}(p)\right) \leq \beta_{*}$ by Lemma 5.2 (iv), we have $G_{\beta}\left(\varphi_{p}\right)>0$, which leads to a contradiction. Therefore, any solution of $\left(D_{\alpha, \beta, f}\right)$ is nonnegative. Moreover, if $f \in L^{\infty}(\Omega) \backslash\{0\}$ and $f \geq 0$ a.e. in $\Omega$, then we have $u \in \operatorname{int} C_{0}^{1}(\bar{\Omega})_{+}$ (see Remark 2.5), that is, $u$ is positive.

Proof of Proposition 2.7 Let us fix some $\beta<\beta_{f}\left(\lambda_{1}(p)\right)$ and consider an arbitrary sequence $\left\{\alpha_{n}\right\}$ such that each $\alpha_{n}>\lambda_{1}(p)$ and $\alpha_{n} \rightarrow \lambda_{1}(p)$ as $n \rightarrow+\infty$. Let $u_{n}$ be a solution of $\left(D_{\alpha, \beta, f}\right)$ with $\alpha=\alpha_{n}$. Assume first that $\left\{u_{n}\right\}$ is bounded in $W_{0}^{1, p}$. Then, we deduce from Remark 2.4 and the Arzelà-Ascoli theorem that $u_{n} \rightarrow u$ in $C^{1}(\bar{\Omega})$, up to a subsequence, where $u$ is a solution of $\left(D_{\alpha, \beta, f}\right)$. Noting that $u \in \operatorname{int} C_{0}^{1}(\bar{\Omega})_{+}$by Theorem 2.6 and Remark 2.5, we conclude that $u_{n} \in \operatorname{int} C_{0}^{1}(\bar{\Omega})_{+}$for all sufficiently large $n \in \mathbb{N}$.

Assume now that $\left\{u_{n}\right\}$ is unbounded in $W_{0}^{1, p}$. Considering the normalized sequence $v_{n}:=$ $u_{n} /\left\|\nabla u_{n}\right\|_{p}$, we can argue as in [7, Lemma 3.3] to obtain that $\left\{v_{n}\right\}$ converges strongly in $W_{0}^{1, p}$ to $\varphi_{p}$ or $-\varphi_{p}$, up to a subsequence. Applying again Remark 2.4 and the Arzelà-Ascoli theorem, we deduce that either $v_{n} \rightarrow \varphi_{p}$ or $v_{n} \rightarrow-\varphi_{p}$ in $C^{1}(\bar{\Omega})$, up to a subsequence. Since $\varphi_{p} \in \operatorname{int} C_{0}^{1}(\bar{\Omega})_{+}\left(\right.$see Remark 2.5), we get either $v_{n} \in \operatorname{int} C_{0}^{1}(\bar{\Omega})_{+}$or $-v_{n} \in \operatorname{int} C_{0}^{1}(\bar{\Omega})_{+}$for all sufficiently large $n \in \mathbb{N}$.

Finally, recalling that the sequence $\left\{\alpha_{n}\right\}$ has been chosen in an arbitrary way, we finish the proof.

Proof of Proposition 2.8 Let $\alpha \geq \lambda_{1}(p)$ and $\beta>\beta_{p s}(\alpha)$. Suppose, by contradiction, that $\left(D_{\alpha, \beta, f}\right)$ has a nonnegative solution $u$ for some $f \in L^{\infty}(\Omega)$ satisfying $f \geq 0$ and $f \not \equiv 0$. Then, $u \in \operatorname{int} C_{0}^{1}(\bar{\Omega})_{+}$(see Remark 2.5), and hence, $u$ is a super-solution of $\left(D_{\alpha, \beta, 0}\right)$. Noting that $\beta>\beta_{p s}(\alpha)$ implies $\beta>\lambda_{1}(q)$ (see [6, Proposition 3 (ii)]) and applying the subsuper-solution method [6, Lemma 6], we obtain a positive solution of $\left(D_{\alpha, \beta, 0}\right)$. However, it contradicts [6, Theorem 2.2] which asserts that $\left(D_{\alpha, \beta, 0}\right)$ has no positive solutions.

Proof of Proposition 2.9 Suppose, by contradiction, that there exists $\alpha>\alpha_{*}$ such that for any $n \in \mathbb{N}$ there exists $\beta_{n}>\lambda_{1}(q)-\frac{1}{n}$ and a nonnegative solution $u_{n}$ of $\left(D_{\alpha, \beta, f}\right)$ with $\beta=\beta_{n}$. 
Since $u_{n} \in \operatorname{int} C_{0}^{1}(\bar{\Omega})_{+}$for each $n \in \mathbb{N}$ (see Remark 2.5), we can choose $\varphi_{q}^{p} / u_{n}^{p-1}$ as a test function for $\left(D_{\alpha, \beta, f}\right)$. Then, we obtain

$$
\begin{aligned}
& \int_{\Omega}\left|\nabla u_{n}\right|^{p-2} \nabla u_{n} \nabla\left(\frac{\varphi_{q}^{p}}{u_{n}^{p-1}}\right) \mathrm{d} x+\int_{\Omega}\left|\nabla u_{n}\right|^{q-2} \nabla u_{n} \nabla\left(\frac{\varphi_{q}^{p}}{u_{n}^{p-1}}\right) \mathrm{d} x \\
& =\alpha \int_{\Omega} \varphi_{q}^{p} \mathrm{~d} x+\beta_{n} \int_{\Omega} \varphi_{q}^{p} u_{n}^{q-p} \mathrm{~d} x+\int_{\Omega} f \frac{\varphi_{q}^{p}}{u_{n}^{p-1}} \mathrm{~d} x .
\end{aligned}
$$

The classical Picone identity (see [2, Theorem 1.1]) implies

$$
\int_{\Omega}\left|\nabla u_{n}\right|^{p-2} \nabla u_{n} \nabla\left(\frac{\varphi_{q}^{p}}{u_{n}^{p-1}}\right) \mathrm{d} x \leq \int_{\Omega}\left|\nabla \varphi_{q}\right|^{p} \mathrm{~d} x=\alpha_{*} \int_{\Omega} \varphi_{q}^{p} \mathrm{~d} x
$$

and the generalized Picone's identity from [16, Lemma 1] yields

$$
\begin{aligned}
\int_{\Omega}\left|\nabla u_{n}\right|^{q-2} \nabla u_{n} \nabla\left(\frac{\varphi_{q}^{p}}{u_{n}^{p-1}}\right) \mathrm{d} x & \leq \int_{\Omega}\left|\nabla \varphi_{q}\right|^{q-2} \nabla \varphi_{q} \nabla\left(\frac{\varphi_{q}^{p-q+1}}{u_{n}^{p-q}}\right) \mathrm{d} x \\
& =\lambda_{1}(q) \int_{\Omega} \varphi_{q}^{p} u_{n}^{q-p} \mathrm{~d} x .
\end{aligned}
$$

Consequently, we get

$$
\left(\alpha-\alpha_{*}\right)\left\|\varphi_{q}\right\|_{p}^{p}+\left(\beta_{n}-\lambda_{1}(q)\right) \int_{\Omega} \varphi_{q}^{p} u_{n}^{q-p} \mathrm{~d} x+\int_{\Omega} f \frac{\varphi_{q}^{p}}{u_{n}^{p-1}} \mathrm{~d} x \leq 0
$$

for each $n \in \mathbb{N}$. Note that the first and third terms in (5.12) are positive, and the first term does not depend on $n$. Thus, if $\beta_{n} \geq \lambda_{1}(q)$ for some $n \in \mathbb{N}$, we get a contradiction. Therefore, we may assume that $\beta_{n} \uparrow \lambda_{1}(q)$, and hence, (5.12) implies

$$
\int_{\Omega} \varphi_{q}^{p} u_{n}^{q-p} \mathrm{~d} x \rightarrow+\infty \text { as } n \rightarrow+\infty
$$

Let us show that this is impossible. Since $\alpha_{*}>\lambda_{1}(p)$ and each $u_{n}>0$, we can argue as in [7, Lemma 3.3] to prove that $\left\{\left\|\nabla u_{n}\right\|_{p}\right\}$ is bounded. Hence, by Remark 2.4 and the Arzelà-Ascoli theorem we obtain that $u_{n} \rightarrow u$ in $C^{1}(\bar{\Omega})$, up to a subsequence, where $u \in \operatorname{int} C_{0}^{1}(\bar{\Omega})_{+}$is a solution of $\left(D_{\alpha, \beta, f}\right)$. Since $\varphi_{q}, u$, and each $u_{n}$ satisfy the boundary point lemma (see, e.g., [27, Theorem 5.5.1]), the $C^{1}(\bar{\Omega})$-convergence implies that we can find $c_{1}, c_{2}>0$ such that

$$
c_{1} \operatorname{dist}(x, \partial \Omega)<\varphi_{q}(x), u(x), u_{n}(x)<c_{2} \operatorname{dist}(x, \partial \Omega)
$$

for any $x \in \Omega$ and all sufficiently large $n \in \mathbb{N}$. Thus, we get

$$
\int_{\Omega} \varphi_{q}^{p} u_{n}^{q-p} \mathrm{~d} x \leq c_{2}^{p} c_{1}^{q-p} \int_{\Omega}(\operatorname{dist}(x, \partial \Omega))^{q} \mathrm{~d} x<+\infty,
$$

which contradicts (5.13).

Let us turn to the proof of Proposition 2.10. We start by showing some basic properties of the critical value $\beta^{f}(\alpha)$ defined by (2.7). Recall that the functional $\Phi_{\alpha}^{-}$and the set $\mathcal{B}^{-}(\alpha)$ are defined by (2.8) and (2.9), respectively.

Lemma 5.4 Let $f \in W^{-1, p^{\prime}}(\Omega) \backslash\{0\}$ and $f \geq 0$ in the weak sense. Then, the following assertions are satisfied: 
(i) $\Phi_{\alpha}^{-}$is 0-homogeneous and $\mathcal{B}^{-}(\alpha) \neq \emptyset$ for any $\alpha \geq \lambda_{1}(p)$;

(ii) $\beta^{f}(\alpha)<+\infty$ for any $\alpha \geq \lambda_{1}(p)$;

(iii) $\beta^{f}$ is nondecreasing in $\left[\lambda_{1}(p),+\infty\right)$;

(iv) $\beta^{f}\left(\lambda_{1}(p)\right)=\beta_{*}$ and $\beta^{f}(\alpha) \rightarrow+\infty$ as $\alpha \rightarrow+\infty$.

Proof Assertion (i) is trivial. Let us prove Assertion (ii). Note that

$$
\mathcal{B}^{-}(\alpha) \subset X(\alpha):=\left\{u \in W_{0}^{1, p}:\|\nabla u\|_{p}^{p} \leq \alpha\|u\|_{p}^{p}\right\}
$$

for all $\alpha \geq \lambda_{1}(p)$, and hence, [29, Lemma 9] implies the existence of a constant $C=C(\alpha)>$ 0 such that

$$
\|\nabla u\|_{p} \leq C\|u\|_{q} \text { for any } u \in \mathcal{B}^{-}(\alpha) .
$$

Applying Hölder's inequality, we get $\|\nabla u\|_{q} \leq|\Omega|^{\frac{p-q}{p q}}\|\nabla u\|_{p}$, which yields the desired boundedness:

$$
\beta^{f}(\alpha) \leq \Phi_{\alpha}^{-}(u) \leq \frac{\|\nabla u\|_{q}^{q}}{\|u\|_{q}^{q}} \leq|\Omega|^{\frac{p-q}{p}} C^{q}<+\infty \text { for any } u \in \mathcal{B}^{-}(\alpha) .
$$

Assertion (iii). We argue similarly to the proof of Proposition 5.2 (v). Suppose, by contradiction, that there exist $\alpha_{1}, \alpha_{2} \geq \lambda_{1}(p)$ such that $\alpha_{1}<\alpha_{2}$ and $\beta^{f}\left(\alpha_{1}\right)>\beta^{f}\left(\alpha_{2}\right)$. That is, we can find $u \in \mathcal{B}^{-}\left(\alpha_{1}\right)$ such that $\Phi_{\alpha_{1}}^{-}(u)>\beta^{f}\left(\alpha_{2}\right)$. Since $H_{\alpha_{1}}(u) \leq 0$, we have $H_{\alpha_{2}}(u)<H_{\alpha_{1}}(u) \leq 0$. Applying Lemma 5.1, we can find a sequence $\left\{u_{n}\right\} \subset W_{0}^{1, p}$ such that $u_{n} \geq 0$ a.e. in $\Omega$,

$$
H_{\alpha_{2}}\left(u_{n}\right)=0 \text { and } \Phi_{\alpha_{2}}^{-}\left(u_{n}\right)=\frac{\left\|\nabla u_{n}\right\|_{q}^{q}}{\left\|u_{n}\right\|_{q}^{q}} \rightarrow \frac{\|\nabla u\|_{q}^{q}}{\|u\|_{q}^{q}} \geq \Phi_{\alpha_{1}}^{-}(u) \text { as } n \rightarrow+\infty \text {. }
$$

We see that $\left\{u_{n}\right\} \subset \mathcal{B}^{+}\left(\alpha_{2}\right)$, and hence, (5.14) leads to a contradiction since $\Phi_{\alpha_{1}}^{-}(u)>$ $\beta^{f}\left(\alpha_{2}\right)$.

Assertion (iv). The equality $\beta^{f}\left(\lambda_{1}(p)\right)=\beta_{*}$ is trivial. To show that $\beta^{f}(\alpha) \rightarrow+\infty$ as $\alpha \rightarrow+\infty$, let us assume, without loss of generality, that $0 \in \Omega$, and let us fix a ball $B \subset \Omega$ such that $0 \in B$. Consider any nonnegative $u \in C_{0}^{\infty}(\Omega)$ and $\alpha \geq \lambda_{1}(p)$ such that supp $u \subset B$ and $\|\nabla u\|_{p}^{p}=\alpha\|u\|_{p}^{p}$, i.e., $H_{\alpha}(u)=0$. Now, we define a function $u_{n}$ by $u_{n}(x)=u(n x)$ for each $n \in \mathbb{N}$. Since supp $u \subset B$, we get $u_{n} \in C_{0}^{\infty}(\Omega)$. Moreover, it is not hard to obtain that

$$
\left\|\nabla u_{n}\right\|_{p}^{p}=\alpha n^{p}\left\|u_{n}\right\|_{p}^{p} \quad \text { and } \quad \Phi_{\alpha n^{p}}^{-}\left(u_{n}\right)=\frac{\left\|\nabla u_{n}\right\|_{q}^{q}}{\left\|u_{n}\right\|_{q}^{q}}=n^{q} \frac{\|\nabla u\|_{q}^{q}}{\|u\|_{q}^{q}} \text {. }
$$

Thus, we see from (5.15) that $u_{n} \in \mathcal{B}^{-}\left(\alpha n^{p}\right)$ and $\beta^{f}\left(\alpha n^{p}\right) \geq \Phi_{\alpha n^{p}}^{-}\left(u_{n}\right) \rightarrow+\infty$ as $n \rightarrow+\infty$.

The following result can be obtained in much the same way as Proposition 5.3.

Proposition 5.5 Let $f \in W^{-1, p^{\prime}}(\Omega)$ and $f \geq 0$ in the weak sense. Assume that $\alpha>\lambda_{1}(p)$ and $\beta>\beta^{f}(\alpha)$. If $u \in W_{0}^{1, p}$ is such that $u \geq 0$ a.e. in $\Omega$ and $H_{\alpha}(u)<0$, then

$$
H_{\alpha}(u)+G_{\beta}(u)-\langle f, u\rangle<0 .
$$

Proof of Proposition 2.10 (i) Let $\beta<\beta_{f}(\alpha)$ and $u^{-} \not \equiv 0$. Suppose, by contradiction, that $H_{\alpha}\left(u^{-}\right) \geq 0$. As in the proof of Theorem 2.6, $-u^{-}$satisfies the equality (5.11). Therefore, if $H_{\alpha}\left(u^{-}\right)>0$, then we get a contradiction to Proposition 5.3 applied to $-u^{-}$. If $H_{\alpha}\left(u^{-}\right)=0$, 
then $-u^{-} \in \mathcal{B}^{+}(\alpha)$, and hence, $\beta<\beta_{f}(\alpha)$ implies $G_{\beta}\left(-u^{-}\right)>0$, which contradicts (5.11). (ii) Let $\beta>\beta^{f}(\alpha)$ and $u^{+} \not \equiv 0$. Suppose, by contradiction, that $H_{\alpha}\left(u^{+}\right) \leq 0$. Since $u$ is a solution of $\left(D_{\alpha, \beta, f}\right)$, we have

$$
\left\langle E_{\alpha, \beta}^{\prime}(u), u^{+}\right\rangle=H_{\alpha}\left(u^{+}\right)+G_{\beta}\left(u^{+}\right)-\left\langle f, u^{+}\right\rangle=0 .
$$

If $H_{\alpha}\left(u^{+}\right)<0$, then we get a contradiction to Proposition 5.5. If $H_{\alpha}\left(u^{+}\right)=0$, then $u^{+} \in$ $\mathcal{B}^{-}(\alpha)$, and hence, $\beta>\beta_{f}(\alpha)$ implies $G_{\beta}\left(u^{+}\right)<0$, which contradicts (5.17). The proof is complete.

Acknowledgements V. Bobkov was supported by the Grant 18-03253S of the Grant Agency of the Czech Republic and by the project LO1506 of the Czech Ministry of Education, Youth and Sports. M. Tanaka was supported by JSPS KAKENHI Grant Number 15K17577. The authors would like to thank the anonymous referee for valuable remarks and suggestions which helped to improve the manuscript.

\section{References}

1. Alama, S., Tarantello, G.: Elliptic problems with nonlinearities indefinite in sign. J. Funct. Anal. 141(1), 159-215 (1996). https://doi.org/10.1006/jfan.1996.0125

2. Allegretto, W., Huang, Y.: A Picone's identity for the $p$-Laplacian and applications. Nonlinear Anal. Theory Methods Appl 32(7), 819-830 (1998). https://doi.org/10.1016/S0362-546X(97)00530-0

3. Ambrosetti, A., Brezis, H., Cerami, G.: Combined effects of concave and convex nonlinearities in some elliptic problems. J. Funct. Anal. 122(2), 519-543 (1994). https://doi.org/10.1006/jfan.1994.1078

4. Anane, A.: Simplicité et isolation de la premiere valeur propre du $p$-laplacien avec poids. Comptes Rendus de l'Académie des Sciences-Series I-Mathematics, 305(16), 725-728 (1987). http://gallica.bnf. fr/ark:/12148/bpt6k574477681/f27

5. Averna, D., Motreanu, D., Tornatore, E.: Existence and asymptotic properties for quasilinear elliptic equations with gradient dependence. Appl. Math. Lett. 61, 102-107 (2016). https://doi.org/10.1016/j. aml.2016.05.009

6. Bobkov, V., Tanaka, M.: On positive solutions for $(p, q)$-Laplace equations with two parameters. Calc. Var. Partial Differ. Equ. 54(3), 3277-3301 (2015). https://doi.org/10.1007/s00526-015-0903-5

7. Bobkov, V., Tanaka, M.: On sign-changing solutions for $(p, q)$-Laplace equations with two parameters. Adv. Nonlinear Anal. (2016). https://doi.org/10.1515/anona-2016-0172

8. Bobkov, V., Tanaka, M.: Remarks on minimizers for $(p, q)$-Laplace equations with two parameters. Commun. Pure Appl. Anal. 17(3), 1219-1253 (2018). https://doi.org/10.3934/cpaa.2018059

9. Chang, K.C.: Infinite dimensional morse theory and multiple solution problems. Birkhäuser (1993). https://doi.org/10.1007/978-1-4612-0385-8

10. Chaves, M.F., Ercole, G., Miyagaki, O.H.: Existence of a nontrivial solution for the ( $p, q)$-Laplacian in $\mathbb{R}^{N}$ without the Ambrosetti-Rabinowitz condition. Nonlinear Anal. Theory Methods Appl. 114, 133-141 (2015). https://doi.org/10.1016/j.na.2014.11.010

11. Clément, P., Peletier, L.A.: An anti-maximum principle for second-order elliptic operators. J. Differ. Equ. 34(2), 218-229 (1979). https://doi.org/10.1016/0022-0396(79)90006-8

12. Drábek, P.: Geometry of the energy functional and the Fredholm alternative for the $p$-Laplacian in higher dimensions. In: Electronic Journal of Differential Equations, Conference 08, 103-120. (2002) https://ejde. math.txstate.edu/conf-proc/08/d1/drabek.pdf

13. Drábek, P., Girg, P., Takáč, P., Ulm, M.: The Fredholm alternative for the $p$-Laplacian: bifurcation from infinity, existence and multiplicity. Indiana Univ. Math. J. 53(2), 433-482. (2004) http://www.jstor.org/ stable/24903516

14. Drábek, P., Robinson, S.B.: Resonance problems for the $p$-Laplacian. J. Funct. Anal. 169(1), 189-200 (1999). https://doi.org/10.1006/jfan.1999.3501

15. Dugundji, J.: An extension of Tietze's theorem. Pac. J. Math. 1(3), 353-367 (1951). https://projecteuclid. org/euclid.pjm/1103052106

16. Il'yasov, Y.: On positive solutions of indefinite elliptic equations. Comptes Rendus de l'Académie des Sciences-Series I-Mathematics 333(6), 533-538 (2001). https://doi.org/10.1016/S0764-4442(01)019243

17. Il'yasov, Y.S.: Bifurcation calculus by the extended functional method. Funct. Anal. Appl. 41(1), 18-30 (2007). https://doi.org/10.1007/s10688-007-0002-2 
18. Filippakis, M.E., Papageorgiou, N.S.: Resonant $(p, q)$-equations with Robin boundary condition. Electron. J. Differ. Equ. 2018(1), 1-24 (2018). https://ejde.math.txstate.edu/Volumes/2018/01/filippakis.pdf

19. Fleckinger, J., Gossez, J.-P., Takáč, P., \& de Thélin, F.: Existence, nonexistence et principe de l'antimaximum pour le $p$-laplacien. Comptes rendus de l'Académie des sciences. Série 1, Mathématique, 321(6), 731-734 (1995) http://gallica.bnf.fr/ark:/12148/bpt6k62037127/f81

20. Fleckinger-Pellé J., Takáč, P.: An improved Poincaré inequality and the $p$-Laplacian at resonance for $p>2$. Adv. Differ. Equ. 7(8), 951-971. http://projecteuclid.org/euclid.ade/1356651685

21. Fučík, S., Nečas, J., Souček, J., Souček, V.: Spectral Analysis of Nonlinear Operators, vol. 346. Springer, New York (2006). https://doi.org/10.1007/BFb0059360

22. Lieberman, G.M.: Boundary regularity for solutions of degenerate elliptic equations. Nonlinear Anal. Theory Methods Appl. 12(11), 1203-1219 (1988). https://doi.org/10.1016/0362-546X(88)90053-3

23. Lieberman, G.M.: The natural generalizationj of the natural conditions of Ladyzhenskaya and Ural'tseva for elliptic equations. Commun. Partial Differ. Equ. 16(2-3), 311-361 (1991). https://doi.org/10.1080/ 03605309108820761

24. Marano, S., Mosconi, S.: Some recent results on the Dirichlet problem for $(p, q)$-Laplace equations. Discrete Contin. Dyn. Syst. Ser. S 11(2), 279-291 (2017). https://doi.org/10.3934/dcdss.2018015

25. Miyajima, S., Motreanu, D., Tanaka, M.: Multiple existence results of solutions for the Neumann problems via super-and sub-solutions. J. Funct. Anal. 262(4), 1921-1953 (2012). https://doi.org/10.1016/j.jfa.2011. 11.028

26. Motreanu, D., Tanaka, M.: On a positive solution for $(p, q)$-Laplace equation with indefinite weight. Minimax Theory Appl. 1, 1-20 (2016). http://www.heldermann-verlag.de/mta/mta01/mta0001-b.pdf

27. Pucci, P., Serrin, J.B.: The Maximum Principle, vol. 73. Springer, New York (2007). https://doi.org/10. 1007/978-3-7643-8145-5

28. Takáč, P.: On the Fredholm alternative for the $p$-Laplacian at the first eigenvalue. Indiana Univ. Math. J. 51(1), 187-238 (2002). https://doi.org/10.1512/iumj.2002.51.2156

29. Tanaka, M.: Generalized eigenvalue problems for $(p, q)$-Laplacian with indefinite weight. J. Math. Anal. Appl. 419(2), 1181-1192 (2014). https://doi.org/10.1016/j.jmaa.2014.05.044

Publisher's Note Springer Nature remains neutral with regard to jurisdictional claims in published maps and institutional affiliations. 